\title{
Partition functions of three-dimensional pure gravity
}

\author{
XI YIN
}

The three-dimensional pure quantum gravity with a negative cosmological constant has been conjectured to be dual to an extremal conformal field theory (ECFT), of central charge $c=24 k$ for some positive integer $k$. We compute the partition function of the dual ECFT by summing over gravitational instanton contributions. In particular, we conjecture an exact expression for the contribution from handlebodies to the partition function for all genera and all values of $k$ and provide non-trivial evidences for the conjecture at genus two.

\section{Introduction}

It is an interesting problem to understand whether three-dimensional pure gravity exists as a quantum theory, and to solve it, if it exists. Witten [1] argued that three-dimensional pure gravity with a negative cosmological constant in $A d S_{3}$ should be dual to an "extremal" conformal field theory (ECFT) on the boundary. The ECFT factorizes into a holomorphic CFT and an anti-holomorphic CFT, and we will mostly consider the holomorphic sector of the CFT. The ECFT has central charge $c=24 k$, where $k$ is a positive integer. So far the only case where the ECFT is known to exist is $k=1$ [2]. Evidences for the existence of $k=2$ ECFT were found in [1] by showing that its partition function on any hyperelliptic Riemann surface can be consistently constructed. It is further shown in [3] that the genus two partition function of the $k=3 \mathrm{ECFT}$ can be consistently constructed as well. ${ }^{1}$

Naturally, one may ask whether the partition function of an ECFT of general $k$, say on a Riemann surface of genus two, can be constructed consistently and whether the answer is unique. One may also wonder to what extent these partition functions can be reproduced from a gravity

\footnotetext{
${ }^{1}$ On the other hand, arguments against the existence of ECFT for large $k$ were presented in [4], based on a conjectural differential equation that constrains the genus one partition function.
} 
computation, assuming that the ECFTs are indeed dual to pure quantum gravity in $A d S_{3}$. In this paper, we will attempt to address both questions and find evidence suggesting that the partition function of the ECFT (when it exists) can be produced exactly from the gravity path integral, as a sum over contributions from gravitational instantons. Moreover, we will conjecture an exact expression for contribution from handlebodies in pure threedimensional gravity of all values of $k$, which is explicitly computable (at least when the Riemann surface is hyperelliptic). Our results suggest that the contributions from the handlebodies could dominate the gravity path integral and are, in particular, responsible for the "polar" part of the full partition function.

This paper is organized as follows. Section 2 gives an overview of our main results and conjectures. The computation of the classical gravity partition function is described in Section 3. In Section 4, we will discuss the $1 / k$ corrections in the gravity computation and relate them to the ECFT partition function. Section 5 generalizes our proposals to higher genera. In Section 6, we describe some possible non-handlebody contributions. Details of the computations, as well as our conventions for Siegel modular forms, Schottky parameterizations and the sewing/cutting of genus two Riemann surfaces, are described in the appendices.

\section{The genus two partition function: gravity vs CFT}

We will now describe some general properties of the genus two partition functions of the $c=24 k$ ECFT, as well as its expected relation to the gravity partition function. The genus two partition function of a CFT with nonzero central charge is subject to the conformal anomaly. When the CFT is holomorphic, we can require the partition function to vary holomorphically with the moduli of the Riemann surface. In the case of genus two, further requiring $\operatorname{Sp}(4, \mathbf{Z})$ modular invariance fixes the partition function up to an overall constant. More precisely, the genus two partition function of the $c=$ $24 k$ ECFT can be regarded as a Siegel modular form of weight $2 k,{ }^{2}$ denoted by $Z_{k, g=2}^{\bmod }(\Omega)$, or $Z_{k}^{\bmod }(\Omega)$ for short. This is the partition function considered in $[1,3]$. One should be cautious that Tuite [5], for instance, uses a different genus two partition function, related to $Z_{k, g=2}^{\bmod }$ by a "holomorphic correction" factor $G(\Omega)^{-k}$, which is not a Siegel modular form. This difference would

\footnotetext{
${ }^{2}$ More precisely, we allow these modular forms to have poles along the divisor in the Siegel upper half space corresponding to the separating degeneration of the Riemann surface; we will loosely call these Siegel modular forms as well.
} 
be important if one studies the factorization of the partition function at the separating degeneration using the $\epsilon$-parameter of [5]. The explicit expression for $Z_{k, g=2}^{\bmod }(\Omega)$ with $k=1$ was obtained in [5], whereas the $k=2,3$ results were computed in [3]. It is of the form

$$
Z_{k, g=2}^{\bmod }(\Omega)=\frac{T_{k}(\Omega)}{\chi_{10}^{k}}
$$

where $\chi_{10}$ is the weight 10 Igusa cusp form, and $T_{k}(\Omega)$ is an entire Siegel modular form of weight $12 k$, given as a polynomial in the generating forms $\psi_{4}, \psi_{6}, \chi_{10}, \chi_{12}$.

The gravity partition function, on the other hand, is computed by summing over saddle point contributions to the path integral. Each saddle point corresponds to a classical solution to the Euclidean equation of motion, i.e., a hyperbolic three-manifold $M$ whose conformal boundary is the given Riemann surface $\Sigma$. In this paper, we will be mostly considering a particularly simple class of such hyperbolic three-manifolds, namely the ones which are handlebodies. ${ }^{3}$ In general, when $\Sigma$ has genus $g>1$, there are a lot of hyperbolic three-manifolds whose conformal boundary is $\Sigma$, which are not handlebodies; ${ }^{4}$ we do not understand their contributions and will comment on them at the end.

The classical instanton action is given by a suitably regularized Einstein-Hilbert action evaluated on $M$. In addition, there are quantum corrections, suppressed by powers of the coupling constant $1 / k$. Higher than 2-loop corrections will vanish if the boundary Riemann surface has genus one, but are in general non-vanishing for higher genus Riemann surfaces (Figure 1).

The contribution from the path integral around a saddle point, corresponding to a handlebody $M$, takes the form

$$
Z_{\text {saddle }}(k, \Omega)=\exp \left[k S_{0}(\Omega)+S_{1}(\Omega)+\frac{1}{k} S_{2}(\Omega)+\frac{1}{k^{2}} S_{3}(\Omega)+\cdots\right],
$$

where $k^{1-l} S_{l}(\Omega)$ is the $l$-loop free energy of the boundary graviton excitations. $\Omega$ is the period matrix of the Riemann surface; in the genus two case,

\footnotetext{
${ }^{3} \mathrm{~A}$ (three-dimensional) handlebody is homeomorphic to the domain enclosed by a closed surface embedded in $\mathbf{R}^{3}$.

${ }^{4}$ I am grateful to E. Witten for pointing this out.
} 


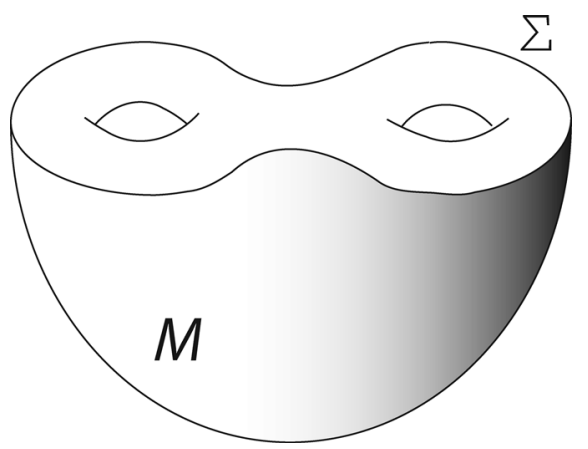

Figure 1: The Riemann surface $\Sigma$ as the conformal boundary of a hyperbolic three-manifold $M$.

we write

$$
\Omega=\left(\begin{array}{ll}
\rho & \nu \\
\nu & \sigma
\end{array}\right)
$$

The tree level (classical) contribution is given by

$$
e^{S_{0}}=\frac{\mathcal{F}(\Omega)^{12}}{\chi_{10}(\Omega)}
$$

where $\mathcal{F}(\Omega)$ is a function of the moduli of the genus two Riemann surface, together with the choice of cycles that are contractible in the hyperbolic three-manifold. Its precise expression will be defined later. We will also conjecture an explicit formula for $S_{1}$.

The contribution from all handlebodies to the full gravity partition function is given by summing over $\operatorname{Sp}(4, \mathbf{Z})$ images of $(2.2)$, with weight $2 k$. We expect $Z_{\text {saddle }}$ to be invariant under $\Gamma_{\infty} \subset \operatorname{Sp}(4, \mathbf{Z})$; this is, in particular, true for $\mathcal{F}(\Omega)$. So the total handlebody contribution is

$$
Z_{h . b .}(k, \Omega)=\sum_{\gamma \in \Gamma_{\infty} \backslash \operatorname{Sp}(4, \mathbf{Z})} \operatorname{det}(C \Omega+D)^{-2 k} Z_{\text {saddle }}(k, \gamma \cdot \Omega) .
$$

The sum in $(2.5)$ converges if $Z_{\text {saddle }}(k, \Omega)$ satisfies suitable regularity conditions and gives an $\operatorname{Sp}(4, \mathbf{Z})$ Siegel modular form. Furthermore, suitable "polar" terms of $Z_{h . b .}(k, \Omega)$ come entirely from the first term in the sum in $(2.5)$, i.e., $Z_{\text {saddle }}(k, \Omega)$ itself. We expect $(2.5)$, together with possible non-handlebody contributions, to give exactly the modular ECFT partition function $Z_{k, g=2}^{\bmod }(\Omega)$. 
It is of interest to compute the $S_{l}$ 's $(l \geq 1)$ directly from loops of boundary graviton excitations. We will however adopt an alternative approach. The idea is that the boundary graviton excitations are nothing but Virasoro descendants of the vacuum, propagating along the handles of the boundary Riemann surface which are filled in by the hyperbolic three-manifold. Let us consider a "fake" CFT partition function

$$
\begin{aligned}
Z_{\text {fake }}(k, \Omega)= & G(\Omega)^{k} \sum_{\mathcal{A}_{i} \in \operatorname{Vir}(k)} \epsilon^{\Delta_{i}-2 k} \operatorname{Tr}_{\operatorname{Vir}(k)}\left(\mathcal{A}_{i} e^{2 \pi i \tau_{1}\left(L_{0}-k\right)}\right) \\
& \times \operatorname{Tr}_{\operatorname{Vir}(k)}\left(\mathcal{A}_{i} e^{2 \pi i \tau_{2}\left(L_{0}-k\right)}\right)
\end{aligned}
$$

where $\tau_{1}$ and $\tau_{2}$ are the moduli of two tori, glued together to form the genus two Riemann surface, with $\epsilon$ being the pinching parameter. The precise definition of $\tau_{1}, \tau_{2}$ and $\epsilon$ are given in [5], which are functions of the period matrix $\Omega$. In the separating degeneration limit $\epsilon \rightarrow 0, \tau_{1}, \tau_{2}, \epsilon$ are approximately $\rho, \sigma, 2 \pi i \nu$. $G(\Omega)$ is the universal "holomorphic correction" factor of [5], needed in relating the torus one-point functions to the modular genus two partition function. The $\mathcal{A}_{i}$ 's in (2.6) run through a set of basis operators in a $c=24 k$ Virasoro algebra, orthonormal with respect to the Zamolodchikov metric. The traces are over all Virasoro descendants of 1 . If $\mathcal{A}_{i}$ 's were to run over all operators in a unitary CFT, and the traces in (2.6) over all operators, (2.6) would have given the modular genus two partition function.

Our main conjecture is that $Z_{\text {fake }}$ is in fact the same as the contribution $Z_{\text {saddle }}$ from the gravitational instanton corresponding to a handlebody. $Z_{\text {fake }}$ is expected to approximate the ECFT partition function in its expansion near $\tau_{1}, \tau_{2} \rightarrow i \infty, \epsilon \rightarrow 0$. In fact, it captures all the terms of $Z_{k}^{\text {mod }}$ that are polar (and constant) in $q=e^{2 \pi i \rho}, s=e^{2 \pi i \sigma}$ (or equivalently, in $e^{2 \pi i \tau_{1}}, e^{2 \pi i \tau_{2}}$ ), since these correspond to the traces in (2.6) over operators with $L_{0} \leq k$, and there are no non-trivial primaries of such weights in the ECFT; $\operatorname{Tr}_{\operatorname{Vir}(k)} \mathcal{A} q^{L_{0}-k}$ would be non-vanishing only if $\mathcal{A}$ is a Virasoro descendant of 1 . In general, such polar terms do not entirely determine $Z_{k}^{\text {mod }}$, since there is an ambiguity of adding to $Z_{k}^{\text {mod }}$ weight $2 k$ cusp forms (i.e., vanishing as $q \rightarrow 0$ or $s \rightarrow 0$ ), for $k \geq 5$. For example, the correlation function of primaries $\left\langle\mathcal{O}_{m} \mathcal{O}_{i} \mathcal{O}_{m}\right\rangle$ contributes to $Z_{k}^{\bmod }(\Omega)$, in the factorization limit, a term of order $e^{2 \pi i\left(\Delta_{m}-k\right)\left(\tau_{1}+\tau_{2}\right)} \epsilon^{\Delta_{i}-2 k} \sim q^{\Delta_{m}-k} s^{\Delta_{m}-k} \nu^{\Delta_{i}-2 k}$. In general, such terms are not fixed by the polar terms and may not be reproduced by summing over $\Gamma_{\infty} \backslash \operatorname{Sp}(4, \mathbf{Z})$ images of $Z_{\text {fake }}$. 
To summarize, our conjectures (in the genus two case) are:

(1) The saddle point contribution $Z_{\text {saddle }}$ in the gravity path integral, corresponding to a handlebody, takes the form (2.2), with $S_{0}$ given by (2.4) (there is also an explicit conjectural formula for $S_{1}$, to be described later.)

(2) $Z_{\text {saddle }}(k, \Omega)=Z_{\text {fake }}(k, \Omega)$ for all $k$, the latter being defined by $(2.6)$ and explicitly computable.

(3) The dual $c=24 k$ ECFT modular genus two partition function $Z_{k}^{\bmod }(\Omega)$ is given by summing over the gravitational instanton contributions, handlebodies as well as non-handlebodies in general. The handlebody contribution $Z_{h . b .}$. (2.5) gives all the polar terms of $Z_{k}^{\text {mod }}$.

These conjectures also admit straightforward generalizations to higher genera. In the remaining sections, we will further explain them and present partial evidences. In Sections 3.4 and 4.1, we will match the terms in $Z_{\text {saddle }}(k, \Omega)$ that are of non-positive powers in $q=e^{2 \pi i \rho}, s=e^{2 \pi i \sigma}$ and to order $\mathcal{O}\left(\nu^{4-2 k}\right)$, with those in $Z_{k}^{\bmod }(\Omega)$, in the cases $k=1,2,3$. Our most non-trivial checks are presented in Section 4.3, in which our conjectured formulae for $S_{0}$ and $S_{1}$ are shown to agree with the leading terms in the $1 / k$ expansion of $\ln Z_{\text {fake }}(k, \Omega)$ up to order $\nu^{4}$.

\section{Classical partition function of pure three-dimensional gravity}

\subsection{Genus one}

We shall consider the geometry of a Euclidean hyperbolic three-manifold with an asymptotic boundary that is conformally equivalent to a torus with complex modulus $\tau$. The regularized Einstein-Hilbert action of this solution is known to be [6]

$$
S=4 \pi k \tau_{2}=-2 \pi i k(\tau-\bar{\tau}),
$$

where $c=24 k=3 l / 2 G, l$ being the curvature radius and $G$ being Newton's constant. The classical limit of the path integral should sum over different ways of filling in the boundary torus, parameterized by $\Gamma_{\infty} \backslash \mathrm{SL}(2, \mathbf{Z})$. So the "naive" genus one partition function is

$$
\sum_{\gamma \in \Gamma_{\infty} \backslash \mathrm{SL}(2, \mathbf{Z})} \exp \left[-2 \pi i k\left(\frac{a \tau+b}{c \tau+d}-\frac{a \bar{\tau}+b}{c \bar{\tau}+d}\right)\right], \quad \gamma=\left(\begin{array}{ll}
a & b \\
c & d
\end{array}\right) .
$$


This result is however in conflict with the assumption of holomorphic factorization. In fact, there appears to be no reason why (3.2) would have an expansion of the form $\sum a_{n, m} q^{n} \bar{q}^{m}$ with integer coefficients $a_{n, m}$, thus in conflict with its interpretation as the partition function of discrete states in $A d S_{3} .{ }^{5}$ We will assume that the left and right moving sectors, corresponding to Chern-Simons gauge fields $A_{\mathrm{L}}$ and $A_{\mathrm{R}}$ of the $\mathrm{SO}(2,1) \times \mathrm{SO}(2,1)$, contribute to the partition function independently. We must then include more general classical Euclidean solutions that involve complex metrics, and the partition function takes the form $\left|Z_{g=1}(\tau)\right|^{2}$, where

$$
Z_{g=1}(\tau)=\sum_{\gamma \in \Gamma_{\infty} \backslash \mathrm{SL}(2, \mathbf{Z})} \exp \left[-2 \pi i k\left(\frac{a \tau+b}{c \tau+d}\right)\right] .
$$

This sum is apparently divergent. It nevertheless can be regularized, as explained in [8], and gives rise to the weakly holomorphic $\operatorname{SL}(2, \mathbf{Z})$ modular form whose only polar term in its $q$-expansion is $q^{-k}$. Up to corrections due to boundary excitations of gravitons (which are one-loop in $1 / k$ ), $Z_{g=1}(\tau)$ coincides with the genus one partition function of the extremal CFT with $c=24 k$.

\subsection{Genus two and higher}

For genus $g>1$, we must compute the regularized Einstein-Hilbert action of the hyperbolic three-manifold $M$ whose conformal boundary is the given genus $g$ Riemann surface $\Sigma_{g}$. In general, $M$ can be constructed as the quotient of hyperbolic three-space $\mathbf{H}_{3}$ by a Kleinian group $\Gamma \subset \operatorname{SL}(2, \mathbf{C})$. When $M$ is a handlebody, the construction is particularly simple; $\Gamma$ will be a Schottky group, i.e., a freely (finitely) generated subgroup of $\mathrm{SL}(2, \mathbf{C})$ that is purely loxodromic. The conformal boundary of $\mathbf{H}_{3}$ is a $\mathbf{C P}^{1} . \Gamma$ is freely generated by $\gamma_{1}, \ldots, \gamma_{g} \in \mathrm{SL}(2, \mathbf{C})$, which act on $\mathbf{C P}^{1}$ as mobius transformations. The quotient of $\mathbf{P}^{1}$ (minus a zero measure limiting set) by $\Gamma$ gives the Riemann surface $\Sigma_{g}$. There are $3 g$ complex parameters for $\gamma_{1}, \ldots, \gamma_{g}$, but they are equivalent to their conjugations by the overall $\mathrm{SL}(2, \mathbf{C})$. So there are $3 g-3$ independent complex parameters, agreeing with the number of complex moduli of the Riemann surface. The $3 g-3$ complex variables parameterizing the generators of $\Gamma$ are coordinates on

\footnotetext{
${ }^{5}$ This is pointed out to me by S. Minwalla. It is also observed by Maloney and Witten [7].
} 


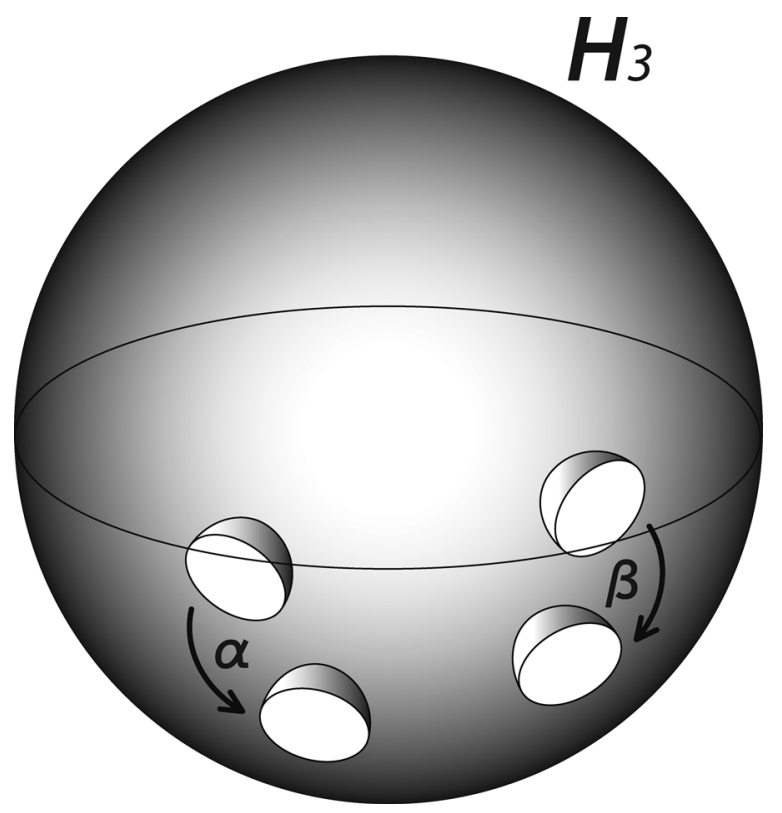

Figure 2: A genus two handlebody $M$ represented as the quotient of hyperbolic three-space $\mathbf{H}_{3}$ by the Schottky group with two generators $\alpha, \beta$. The shaded region (outside the four hemispheres) is a fundamental domain for $M$.

the Schottky space, which is a covering space of the moduli space of $\Sigma_{g}$ (Figure 2).

It is shown in [9] that the regularized Einstein-Hilbert action evaluated on the hyperbolic three-manifold is given by a suitably defined classical Liouville action $S_{\mathrm{L}}[\phi]$ evaluated at its critical point (i.e., $d s^{2}=e^{\phi} d z d \bar{z}$ being the uniformizing metric), whose value we denote by $S_{\mathrm{L}}$. An important point is that the Liouville action cannot be defined by naively integrating the Liouville Lagrangian density $\mathcal{L}=(\partial \phi)^{2}+e^{\phi}$ over the entire Riemann surface, as $\mathcal{L}$ does not transform covariantly under coordinate transformations between differences patches of the Riemann surface. The way to fix this is to define the Liouville action by integrating the Lagrangian density over a fundamental domain in $\mathbf{C}$ that parameterizes the Riemann surface $\Sigma_{g}$, with suitable boundary terms included [10].

There are two standard parameterizations of $\Sigma_{g}$, the Schottky parameterization and Fuchsian parameterization. The former, as described earlier, models $\Sigma_{g}$ as a quotient of the complex plane $\mathbf{C}$ (or $\mathbf{P}^{1}$ ) by the Schottky group $\Gamma \subset \mathrm{SL}(2, \mathbf{C})$. The latter models $\Sigma_{g}$ as the quotient of the hyperbolic 
plane by a discrete subgroup of $\operatorname{SL}(2, \mathbf{R})$. It was shown in [10] that the Liouville action defined using the Schottky parameterization evaluates at its critical point to a Kähler potential of the Weil-Petersson metric on the Schottky space, whereas the action defined in Fuchsian parameterization evaluates to a trivial constant proportional to $2 g-2$. We are looking for a suitably defined $S_{\mathrm{L}}$ that is the sum of a holomorphic function and an antiholomorphic function in the moduli, and neither of these could serve as the classical saddle point contribution in three-dimensional pure gravity.

A hint comes from the following formula for the holomorphic factorization of the determinant of the scalar Laplacian on $\Sigma_{g}$, due to Zograf, and was described in [11]:

$$
\frac{\operatorname{det} \Delta}{\operatorname{det} \operatorname{Im} \Omega}=c_{g} e^{-1 / 12 S_{\mathrm{L}}(\Omega)}|\mathcal{F}(\Omega)|^{2} .
$$

On the RHS of (3.4), $c_{g}$ is a constant that depends on $g$ only. In the context of [11], the determinant of the Laplacian is defined using zeta function regularization in the uniformizing hyperbolic metric on $\Sigma_{g}$ (with constant curvature -1 ), and $S_{\mathrm{L}}(\Omega)$ is the Liouville action defined in Schottky parameterization. $\mathcal{F}(\Omega)$ is a holomorphic function on the Schottky space, given by the following infinite product formula

$$
\mathcal{F}(\Omega)=\prod_{\gamma} \prod_{\text {prim. }}^{\infty}\left(1-q_{\gamma}^{m}\right),
$$

where the first product runs over primitive conjugacy classes of the Schottky group $\Gamma=\left\langle\gamma_{1}, \ldots, \gamma_{g}\right\rangle$. Primitive here means that $\gamma$ is not a positive power of any other element in $\Gamma . q_{\gamma}$ is defined as follows: every element $\gamma \in \Gamma$ is (uniquely) conjugate under $\mathrm{SL}(2, \mathbf{C})$ to $z \mapsto \lambda z$, with $|\lambda|<1$, and $q_{\gamma} \equiv \lambda$. More explicitly,

$$
\frac{\gamma(z)-\eta}{\gamma(z)-\xi}=q_{\gamma} \frac{z-\eta}{z-\xi},
$$

where $\xi, \eta$ are fixed points of $\gamma$. Since the Schottky group elements $\gamma$ in fact only depend on the period matrix $\Omega$ modulo integral shifts, $q_{\gamma}$ are functions of $\left\{e^{2 \pi i \Omega_{m n}}\right\}$ (but they are not simply products of $e^{2 \pi i \Omega_{m n}}$ ).

To be explicit, let us restrict to the genus two case. In general, one expects an ambiguous factor in the determinant of the Laplacian due to the conformal anomaly - there is no canonical choice of the scale factor of the metric on the conformal boundary $\Sigma_{g}$; but this is the same kind of ambiguity 
that appears in $S_{\mathrm{L}}$. To determine the Liouville action $S_{\mathrm{L}}$ corresponding to the classical gravitational instanton action, consider the modular partition function of a single free boson,

$$
(\operatorname{det} \Delta)^{-1 / 2}=(\operatorname{det} \operatorname{Im} \Omega)^{-1 / 2}\left|\chi_{10}(\Omega)\right|^{-1 / 12} .
$$

Plugging (3.7) into (3.4), we obtain a holomorphically factorized "Liouville action" $S_{\mathrm{L}}=S_{\mathrm{hol}}+\overline{S_{\mathrm{hol}}}$, with

$$
e^{-1 / 12 S_{\mathrm{hol}}}=\chi_{10}(\Omega)^{1 / 12} \mathcal{F}(\Omega)^{-1}
$$

The holomorphic part of the Euclidean action for pure three-dimensional gravity of $c=24 k$ should then be identified with

$$
e^{k S_{\mathrm{hol}}}=\left[\frac{\mathcal{F}(\Omega)^{12}}{\chi_{10}(\Omega)}\right]^{k}
$$

In the end, we want to sum over all inequivalent $\operatorname{Sp}(4, \mathbf{Z})$ images of (3.9), which should produce the contribution to the partition function from all handlebodies, up to loop corrections suppressed by $1 / k$. In general, the handlebody filling in a Riemann surface $\Sigma_{g}$ is invariant under $\Gamma_{\infty} \subset \operatorname{Sp}(2 g, \mathbf{Z})$, the subgroup of $\operatorname{Sp}(2 g, \mathbf{Z})$ that fixes the cusp $\Omega=i \infty \cdot \mathbf{1}$. Equivalently, $\Gamma_{\infty}$ consisting of elements of the form

$$
\gamma=\left(\begin{array}{cc}
A & B \\
0 & D
\end{array}\right) \in \operatorname{Sp}(2 g, \mathbf{Z}), \quad A D^{\mathrm{T}}=1, \quad A B^{\mathrm{T}}=B A^{\mathrm{T}} .
$$

Thus, we expect $\mathcal{F}(\Omega)$ to be invariant under $\Gamma_{\infty}$. This is indeed the case, as shown in Appendix B.

\subsection{The expansion of $\mathcal{F}(\Omega)$}

The expression (3.5) for $\mathcal{F}$ is rather complicated. In practice, we would like to expand $\mathcal{F}$ near the separating degeneration limit $\nu \rightarrow 0$ and compare with the corresponding expansion of a Siegel modular form. First, to compute $q_{\gamma}$, one must express the Schottky parameters in terms of the periods $\rho, \sigma, \nu$. An explicit formula for this is given in [12] and is recalled in Appendix B. Next, 
one can order the conjagacy classes $\gamma$ according to the order of $q_{\gamma}$ in $\nu$. The primitive conjugacy classes are

$$
\begin{gathered}
\alpha^{ \pm 1}, \quad \beta^{ \pm 1}, \quad \alpha^{n} \beta^{m}(n, m \neq 0), \\
\alpha^{n} \beta^{m} \alpha^{n^{\prime}} \beta^{m^{\prime}}\left((n, m)<\left(n^{\prime}, m^{\prime}\right)\right), \ldots,
\end{gathered}
$$

where for $\alpha^{n} \beta^{m} \alpha^{n^{\prime}} \beta^{m^{\prime}}$ we must choose $(n, m)$ to be distinct from $\left(n^{\prime}, m^{\prime}\right)$; and interchanging $(n, m)$ with $\left(n^{\prime}, m^{\prime}\right)$ gives the same conjugacy classes. In the $\nu \rightarrow 0$ limit, we shall find

$$
q_{\alpha^{\#}}, q_{\beta^{\#}} \sim \mathcal{O}(1), \quad q_{\alpha^{\#} \beta^{\#}} \sim \nu^{2}, \quad q_{\alpha^{\#} \beta^{\#} \alpha^{\#} \beta^{\#}} \sim \nu^{4}, \ldots,
$$

where \# stands for arbitrary non-zero integers. Up to order $\nu^{4}$, it suffices to take into account only the conjugacy classes in (3.11) in the product formula for $\mathcal{F}(\Omega)$. The details of the computation can be found in Appendix C. The result is

$$
\begin{aligned}
\mathcal{F}(\Omega)= & \prod_{m=1}^{\infty}\left(1-q^{m}\right)^{2}\left(1-s^{m}\right)^{2}\left\{1+(2 \pi i \nu)^{2} 4 \hat{E}_{2}^{\rho} \hat{E}_{2}^{\sigma}\right. \\
& +\frac{(2 \pi i \nu)^{4}}{3}\left[-2\left(\hat{E}_{2}^{\rho}\right)^{2} \hat{E}_{2}^{\sigma}-2 \hat{E}_{2}^{\rho}\left(\hat{E}_{2}^{\sigma}\right)^{2}+48\left(\hat{E}_{2}^{\rho}\right)^{2}\left(\hat{E}_{2}^{\sigma}\right)^{2}\right. \\
& \left.\left.-10\left(\hat{E}_{2}^{\rho}\right)^{2} \hat{E}_{4}^{\sigma}-10\left(\hat{E}_{2}^{\sigma}\right)^{2} \hat{E}_{4}^{\rho}-5 \hat{E}_{4}^{\rho} \hat{E}_{4}^{\sigma}\right]+\mathcal{O}\left(\nu^{6}\right)\right\}
\end{aligned}
$$

where $\hat{E}_{n}^{\rho}$ is the $n$th Eisenstein series $E_{n}(\rho)$ with the constant term subtracted, and normalized so that its $q$-expansion starts with $q+\cdots$. In other words, $\hat{E}_{n}^{\rho}=\sum_{m=1}^{\infty} m^{n-1} q^{m} /\left(1-q^{m}\right)$, and similarly for $\hat{E}_{n}^{\sigma}$.

\subsection{Series expansion of the ECFT genus two partition functions}

In order to compare the classical gravity partition function with the known ECFT genus two partition functions, we shall consider the Laurent expansion of the latter in $q, s$ and $\nu . Z_{k, g=2}^{\bmod }$ with $k=1,2,3$ are given explicitly in terms of the generating Siegel modular forms $\psi_{4}, \psi_{6}, \chi_{10}, \chi_{12}$ in $[3,5]$. The generating forms can be expressed in terms of products and/or sums of 
the 10 weight $1 / 2$ characteristic theta series, as in Appendix A. We find the following expansions of the $T_{k}(\Omega)$ 's (defined in (2.1)), to order $\left(q^{k}, s^{k}, \nu^{4}\right)$ :

$$
\begin{aligned}
T_{1}(\Omega)= & (1-24 q)(1-24 s)+48 q s(2 \pi i \nu)^{2}-20 q s(2 \pi i \nu)^{4}+\mathcal{O}\left(q^{2}, s^{2}, \nu^{6}\right) \\
T_{2}(\Omega)= & \left(1-48 q+1081 q^{2}\right)\left(1-48 s+1081 s^{2}\right) \\
& +\frac{q s(-24+1081 q)(-24+1081 s)}{6}\left(2 \pi i \nu^{2}\right) \\
& +\frac{q s[-2880+105384(q+s)-3478739 q s]}{72}(2 \pi i \nu)^{4}+\mathcal{O}\left(q^{3}, s^{3}, \nu^{6}\right) \\
T_{3}(\Omega)= & \left(1-72 q+2485 q^{2}-54599 q^{3}\right)\left(1-72 s+2485 s^{2}-54599 s^{3}\right) \\
(3.14) \quad & +\frac{q s\left(-72+4970 q-163797 q^{2}\right)\left(-72+4970 s-163797 s^{2}\right)}{36}(2 \pi i \nu)^{2} \\
& +q s\left[-60+\frac{10907}{3}(q+s)-\frac{204729}{2}\left(q^{2}+s^{2}\right)-\frac{22571123}{108} q s\right. \\
& \left.+\frac{130929005}{24} q s(q+s)-\frac{2004085311}{16} q^{2} s^{2}\right](2 \pi i \nu)^{4}+\mathcal{O}\left(q^{4}, s^{4}, \nu^{6}\right) .
\end{aligned}
$$

The higher order terms in $q$ and $s$ are non-polar and are not expected to agree

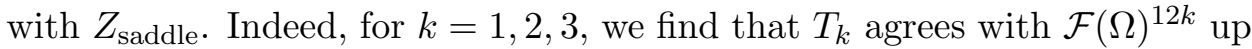
to terms of order $\mathcal{O}\left(q^{2}, s^{2}, \nu^{6}\right)$. In the case $k=1$, this is all the agreement one could hope for. For $k=2,3$, at order $q^{2}, s^{2}$ and higher, one must include the $1 / k$ loop corrections $S_{1}, S_{2}, \ldots$, as will be discussed in the next section.

\section{Going beyond the classical level}

\subsection{The loop expansion in $1 / k$}

We expect that the full (perturbative) contribution around a saddle point, $Z_{\text {saddle }}$, corresponding to a particular filling hyperbolic three-manifold $M$, takes the form (2.2). When $M$ is a handlebody, $Z_{\text {saddle }}$ should be invariant under $\Gamma_{\infty}$, and the summation over $\operatorname{Sp}(4, \mathbf{Z}) / \Gamma_{\infty}$ images of $(2.2)$ then account for all handlebody instanton contributions. The bulk gravity Lagrangian reduces to that of $\mathrm{SL}(2, \mathbf{R})$ WZW model on the boundary Riemann surface [13], which factorizes into a chiral part and an anti-chiral part. In principle, one should compute the $l$-loop free energy of the $\operatorname{SL}(2, \mathbf{R})$ 
WZW model on the genus two Riemann surface, which is expected to give the term $k^{1-l} S_{l}$ in (2.2). We will describe an alternative approach to compute the loop corrections in the next subsection.

Motivated by the product formula for the one-loop free energy of Liouville theory on a general Riemann surface [14], we conjecture the following "holomorphic version" of the product formula as the one-loop free energy of the chiral SL $(2, \mathbf{R})$ WZW model

$$
e^{S_{1}}=\prod_{\gamma \text { prim. }} \prod_{m=2}^{\infty}\left(1-q_{\gamma}^{m}\right)^{-1 / 2} .
$$

Note that the product in (4.1) is similar to the one in (3.5), but with a different range of $m$, and raised to the power $-1 / 2$. The power $1 / 2$ is due to our convention, counting $\gamma$ and $\gamma^{-1}$ as distinct primitive classes. It is conceivable that (4.1) has the interpretation as the determinant of a $\bar{\partial}$-like operator on the Riemann surface, and it would be nice to understand this. Another check of (4.1) is that it is consistent with the genus one answer in the factorization limit and is manifestly $\Gamma_{\infty}$ invariant.

Up to order $\nu^{4},(4.1)$ can be calculated using the expansions of $q_{\alpha}, q_{\beta}$ and $q_{\alpha^{n} \beta^{m}}$ computed earlier. Explicitly, we have

$$
\begin{aligned}
e^{S_{1}}= & \prod_{n=2}^{\infty}\left(1-q^{n}\right)^{-1}\left(1-s^{n}\right)^{-1} \times[1-2 q s(q+s+3 q s)(2+3 q+3 s+8 q s) \\
4.2) \quad & \times(2 \pi i \nu)^{2}+\frac{q s}{6}\left(-2(q+s)+45\left(q^{2}+s^{2}\right)+72 q s+745\left(q^{2} s+q s^{2}\right)\right. \\
& \left.\left.+3720 q^{2} s^{2}\right)(2 \pi i \nu)^{4}+\mathcal{O}\left(q^{4}, s^{4}, \nu^{6}\right)\right] .
\end{aligned}
$$

As remarked at the end of Section $3, S_{1}, S_{2}, \ldots$ are needed in $Z_{\text {saddle }}(k, \Omega)$ in order to compare with the polar terms in $Z_{k}^{\bmod }(\Omega)$ for $k=2,3$. Let us compare $\chi_{10}^{k} Z_{\text {saddle }}(k, \Omega)$ with $T_{k}(\Omega)(3.14)$. At order $\nu^{0}$, the agreement is a trivial consequence of the genus one result and the factorization of $Z_{k}^{\bmod }(\Omega)$. At order $\nu^{2}$, only one- and two-loop corrections, i.e., $S_{1}, S_{2}$, in addition to $S_{0}$, are involved in $Z_{\text {saddle }}$. Having the explicit (conjectured) expressions for $S_{0}(3.13)$ and $S_{1}(4.2)$, the matching of $Z_{\text {saddle }}(k=2)$ with the polar terms in $Z_{k=2}^{\text {mod }}$ already fixes $S_{2}$ at order $\nu^{2}$, up to $q^{2}, s^{2}$. Further requiring $Z_{\text {saddle }}(k=3)$ to agree with $Z_{k=3}^{\bmod }$ then provides a non-trivial check for the expression of $S_{2}$ up to order $q^{2} s^{2} \nu^{2}$. This is indeed the case. Using the comparison with the polar terms in the $k=3$ case, we can further determine $S_{2}$ up to order $q^{3} s^{3} \nu^{2}$. 
At order $\nu^{4}$, we expect $S_{1}, S_{2}, S_{3}$ to contribute to $\chi_{10}^{k} Z_{\text {saddle }}{ }^{6}$ By comparing with up to order $q^{2} s^{2} \nu^{4}$ terms in $T_{2}(\Omega)$ and $T_{3}(\Omega)$ (which are polar), we can determine the order $q^{2} s^{2} \nu^{4}$ terms in $S_{2}$ and $S_{3}$. The result is summarized in the following expansion:

$$
\begin{aligned}
S_{2}(\Omega)= & q^{2} s^{2}\left(\frac{1}{3}+\frac{1}{2} q+\frac{1}{2} s+\frac{3}{4} q s+\cdots\right)(2 \pi i \nu)^{2} \\
& +q^{2} s^{2}\left(\frac{13}{36}+\frac{1}{8}(q+s)-\frac{45}{16} q s+\cdots\right)(2 \pi i \nu)^{4}+\mathcal{O}\left(\nu^{6}\right), \\
S_{3}(\Omega)= & \mathcal{O}\left(q^{4} s^{4} \nu^{4}, \nu^{6}\right) .
\end{aligned}
$$

It would be interesting to reproduce (4.3) directly from perturbative computations in the chiral $\operatorname{SL}(2, \mathbf{R})$ WZW model.

\subsection{The "fake" CFT on the Riemann surface}

The genus one expression for $Z_{\text {saddle }}$

$$
q^{-k} \prod_{n=2}^{\infty}\left(1-q^{n}\right)^{-1}
$$

which is valid to all-loop in $1 / k$, suggests that the only states propagating along handles of the Riemann surface are Virasoro descendants of the ground state. We are led to hypothesize that $Z_{\text {saddle }}$ can be computed by pretending that we have a $c=24 k$ (non-unitary) CFT on the boundary Riemann surface, whose only operators are Virasoro descendants of the identity, namely $T, \partial T, \ldots$ The genus two partition function $Z_{\text {fake }}$ of this "fake" CFT can be computed by gluing the four-point functions of all Virasoro descendants on the sphere. Equivalently, by the factorization of tree level four-point function into three-point functions, $Z_{\text {fake }}$ is given by (2.6) (Figure 3).

An alternative factorization of the four-point function of the Virasoro descendants gives a representation of $Z_{\text {fake }}$ of the form

$$
Z_{\text {fake }}=\tilde{G}(\Omega)^{k} \sum_{\mathcal{A}_{i}, \mathcal{A}_{j}, \mathcal{A}_{l} \in \operatorname{Vir}(k)}\left\langle\mathcal{A}_{i} \mathcal{A}_{j} \mathcal{A}_{l}\right\rangle \tilde{q}_{1}^{\Delta_{i}-k} \tilde{q}_{2}^{\Delta_{j}-k} \tilde{q}_{3}^{\Delta_{l}-k}\left\langle\mathcal{A}_{i} \mathcal{A}_{j} \mathcal{A}_{l}\right\rangle
$$

where $\tilde{G}(\Omega)$ is another possible universal "holomorphic correction" factor, and $\tilde{q}_{i}=e^{2 \pi i \tilde{\tau}_{i}}(i=1,2,3)$ are determined by the moduli of the genus two

\footnotetext{
${ }^{6}$ In fact, we expect all $S_{l}$ 's to contribute at this order. However, it appears that the contributions from $S_{l \geq 4}$ are of higher order in $q, s$.
} 


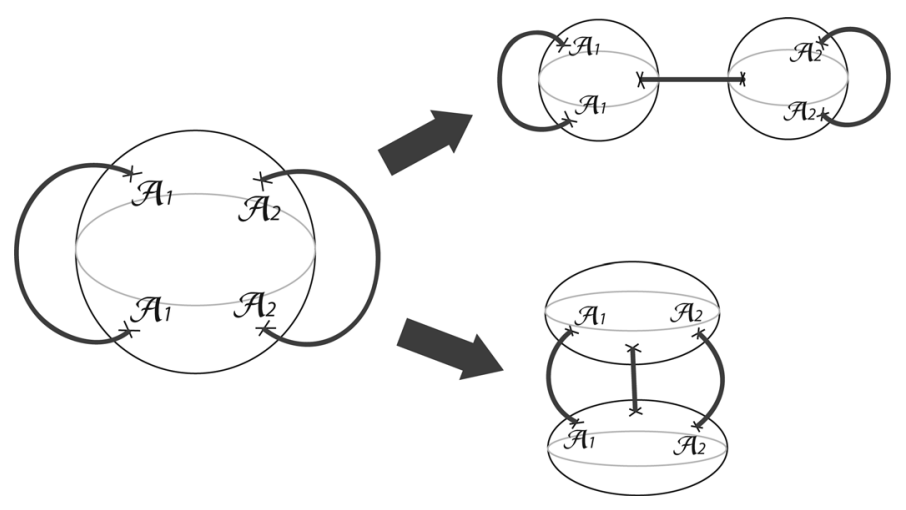

Figure 3: The fake genus two partition function is obtained by gluing sphere four-point functions of Virasoro descendants of the identity. It can be factorized into three-point functions in two different ways.

Riemann surface. Another way to write (4.5) is to express the fake partition function in terms of twist fields in the 2-fold symmetric product of the "fake" CFT, analogously to [1]. In a general 2-fold symmetric product CFT, the twist field $\mathcal{E}$ has OPE with itself of the form

$$
\begin{aligned}
\mathcal{E}(x / 2) \mathcal{E}(-x / 2) & =x^{-3 k} \Psi_{x}(0)+(\text { primaries and their descendants }), \\
\Psi_{x} & =\sum_{i, j} x^{\Delta_{i}+\Delta_{j}} c_{i j} \mathcal{A}_{i}^{+} \mathcal{A}_{j}^{-}
\end{aligned}
$$

where $\mathcal{A}_{i}^{+}$and $\mathcal{A}_{i}^{-}$are Virasoro descendants of the identity (of dimension $\Delta_{i}$ ) in the two copies of the CFT, and $c_{i j}$ are constant coefficients. The genus two Riemann surface is represented as the hyperelliptic curve $y^{2}=\prod_{i=1}^{6}\left(x-e_{i}\right)$. An explicit formula for $\Psi_{x}$ up to order $\mathcal{O}\left(x^{8}\right)$ is given in [3]. We shall choose the branch cuts connecting $\left(e_{1}, e_{2}\right),\left(e_{3}, e_{4}\right),\left(e_{5}, e_{6}\right)$, to correspond to the three "filled" handles, respectively. The fake partition function is given by the six-point function of $\mathcal{E}\left(e_{i}\right)$ 's, but dropping the contributions from primaries in the $\mathcal{E} \mathcal{E}$ OPE (4.6) of nearby branch points, namely the OPEs $\mathcal{E}\left(e_{1}\right) \mathcal{E}\left(e_{2}\right), \mathcal{E}\left(e_{3}\right) \mathcal{E}\left(e_{4}\right)$ and $\mathcal{E}\left(e_{5}\right) \mathcal{E}\left(e_{6}\right)$. Note that $x^{-3 k} \Psi_{x}(0)$ transforms in the same way as $\mathcal{E}(x / 2) \mathcal{E}(-x / 2)$ under conformal transformations. Explicitly, we can write an $\operatorname{SL}(2, \mathbf{C})$ covariant expression $\tilde{Z}_{\text {fake }}$,

$$
\tilde{Z}_{\text {fake }}\left(e_{1}, e_{2}, \ldots, e_{6}\right)=\prod_{1 \leq i<j \leq 6} e_{i j}^{k} \frac{\left\langle\Psi_{e_{12}}\left(\frac{e_{1}+e_{2}}{2}\right) \Psi_{e_{34}}\left(\frac{e_{3}+e_{4}}{2}\right) \Psi_{e_{56}}\left(\frac{e_{5}+e_{6}}{2}\right)\right\rangle}{\left(e_{12} e_{34} e_{56}\right)^{3 k}} .
$$


Under the $\operatorname{SL}(2, \mathbf{C})$ action on the $e_{i}$ 's, $e_{i} \rightarrow\left(a e_{i}+b\right) /\left(c e_{i}+d\right), \tilde{Z}_{\text {fake }}$ transforms covariantly with weight $-2 k$ :

$$
\tilde{Z}_{\text {fake }} \longrightarrow \tilde{Z}_{\text {fake }} \prod_{i=1}^{6}\left(c e_{i}+d\right)^{-2 k} .
$$

The period matrix $\Omega$ is determined by the $e_{i}$ 's up to the overall $\mathrm{SL}(2, \mathbf{C})$ action. They can be mapped to the generating Siegel modular forms via the formulae in [15], as recalled in [3]. The fake partition function $Z_{\mathrm{fake}}(k, \Omega)$ as a function of the periods can be recovered from $\tilde{Z}_{\text {fake }}$ as

$$
Z_{\text {fake }}(k, \Omega) \propto\left[\int_{\alpha_{1}} \frac{d x}{y} \int_{\alpha_{2}} \frac{x d x}{y}-\left(\alpha_{1} \leftrightarrow \alpha_{2}\right)\right]^{2 k} \tilde{Z}_{\text {fake }}\left(e_{1}, \ldots, e_{6}\right),
$$

where $\alpha_{1}$ and $\alpha_{2}$ are a pair of basis $A$-cycles. Note that the RHS of (4.9) is $\operatorname{SL}(2, \mathbf{C})$ invariant. The modular group $\operatorname{Sp}(4, \mathbf{Z})$ acts as monodromies that permute the $e_{i}$ 's. Note that (4.7) involves an infinite series of rational functions of the $e_{i}$ 's, and we expect $Z_{\text {fake }}$ to have branch cuts in the $e_{i}$ 's; it should be invariant under $\Gamma_{\infty}$ only. One can use either (2.6) or (4.7) to compute $Z_{\text {fake }}(k, \Omega)$ explicitly, order by order. We will use the former in the explicit comparison of $Z_{\text {fake }}$ with $S_{0}, S_{1}$ below.

In the cases $k=1,2,3$, by the construction of [3], the polar terms of $Z_{\text {fake }}(k, \Omega)$ automatically agrees with those of $Z_{k}^{\bmod }(\Omega)$. So the checks in the previous subsection amount to the statement that the polar terms of $Z_{\text {saddle }}(k, \Omega)$ agrees with those of $Z_{\text {fake }}(k, \Omega)$. We will show in the next subsection that our conjectured formula for $S_{0}$ and $S_{1}$ in $Z_{\text {saddle }}$, remarkably, agrees with $Z_{\text {fake }}$ up to order $\nu^{4}$ in the $\nu \rightarrow 0$ limit, for all $k$. This lead us to conjecture that $Z_{\text {fake }}(k, \Omega)=Z_{\text {saddle }}(k, \Omega)$. In particular, (2.4) and (4.1) are then predictions for the leading terms in the large $k$ limit of $Z_{\text {fake }}(k, \Omega)$ !

Finally, we conjecture the following formula for the contribution from all handlebody geometries in the pure three-dimensional gravity,

$$
\sum_{\gamma \in \Gamma_{\infty} \backslash \operatorname{Sp}(4, \mathbf{Z})} \operatorname{det}(C \Omega+D)^{-2 k} Z_{\text {fake }}(k, \gamma \cdot \Omega)=Z_{h . b .}(k, \Omega)
$$

And $Z_{h . b .}(k, \Omega)$ appears to capture at least all the polar terms in the partition function $Z_{k}^{\bmod }(\Omega)$ of the dual ECFT, if the latter exists. A few comments are in order. First, in the expansion of $Z_{\text {fake }}$ in terms of (fake) torus one-point functions (2.6), terms that are polar and constant in both $q_{1}$ and $q_{2}$ in $Z_{\text {fake }}$ must agree with $Z_{k}^{\text {mod }}$, as explained in Section 2. In other words, $Z_{k}^{\text {mod }}=$ $Z_{\text {fake }}+\mathcal{O}\left(q_{1}, q_{2}\right)$. In Appendix A.2, we show that with certain assumptions 
on the regularity of $Z_{\text {fake }}(k, \Omega)$ (away from its obvious singularities), the LHS of (4.10) agrees with $Z_{\text {fake }}$ itself at least up to terms of order $\mathcal{O}\left(q_{1}^{0}, q_{2}^{0}\right)$. This is consistent with the expected property of the dual ECFT.

Another check of our proposal is in the limit where three handles of the Riemann surface are pinched, and $Z_{\text {fake }}$ can be expanded as (4.7), with $e_{12}, e_{34}, e_{56} \rightarrow 0$. A subleading term in the Poincaré series (4.10), i.e., one with $\gamma \notin \Gamma_{\infty}$, is given by (4.7) with certain monodromies on the $e_{i}$ 's (we expect that there are branch cuts). After such a permutation, at most one of $e_{12}, e_{34}, e_{56}$ may approach zero in the pinching limit. It is conceivable that the factor $\langle\Psi \Psi \Psi\rangle$ in (4.7) does not give extra singularities in this limit. In the representation (4.5), we then conclude that the terms in Poincare series of $Z_{\text {fake }}$ that are polar in a pair of $\tilde{q}_{i}$ 's must agree with those in $Z_{\text {fake. }}$ This is consistent with what we expect from $Z_{k}^{\mathrm{mod}}$, since terms that are polar in a pair of $\tilde{q}_{i}$ 's come from sewing together sphere three-point functions involving at least two Virasoro descendants of the identity, and hence must agree with $Z_{\text {fake }}$.

A priori, one expects $Z_{k}^{\text {mod }}$ to receive contributions from non-handlebody geometries as well, in addition to the handlebody contribution $Z_{h . b .}$. How-

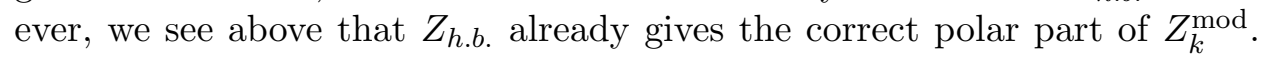
It is then plausible that the non-handlebody geometries only contribute to the non-polar part of $Z_{k}^{\text {mod }}$. Curiously, when $k=1$, the genus two partition function has weight 2 , and there are no weight 2 entire Siegel modular forms (i.e., with no singularities). This means that $Z_{h . b .}(1, \Omega)$ as given by (4.10) is exactly equal to $Z_{k=1}^{\bmod }(\Omega)$. It would be interesting to understand why the non-handlebody contributions would cancel in this case, and if such cancelation could happen for other values of $k$ as well.

\subsection{Comparing the factorization of $Z_{\text {fake }}$ with $Z_{\text {saddle }}$}

Let us compute $Z_{\text {fake }}$ near the separating degeneration, up to order $\nu^{4}$. It is given by

$Z_{\text {fake }}(k, \Omega)=G(\Omega)^{k} \epsilon^{-2 k}\left[Z_{\mathrm{Vir}}\left(\tau_{1}\right) Z_{\mathrm{Vir}}\left(\tau_{2}\right)+\frac{\epsilon^{2}}{12 k} \frac{1}{2 \pi i} \partial_{\tau_{1}} Z_{\mathrm{Vir}}\left(\tau_{1}\right)\right.$

$$
\left.\times \frac{1}{2 \pi i} \partial_{\tau_{1}} Z_{\mathrm{Vir}}\left(\tau_{1}\right)+\frac{5 \epsilon^{4}}{24 k(60 k+11)}\langle T * T\rangle_{\tau_{1}}^{\prime}\langle T * T\rangle_{\tau_{2}}^{\prime}+\mathcal{O}\left(\epsilon^{6}\right)\right],
$$

where the relation between $\tau_{1}, \tau_{2}, \epsilon$ and $\rho, \sigma, \nu$, as well as the function $G(\Omega)$, are described in [5] and recalled in Appendix D. $Z_{\mathrm{Vir}}(\tau)=q^{-k} \prod_{n=2}^{\infty}$ $\left(1-q^{n}\right)^{-1}$ is the Virasoro character. $\langle\cdots\rangle_{\tau}^{\prime}$ stands for the torus one-point 
function in the fake CFT. $T * T(0) \equiv \operatorname{Res}_{z \rightarrow 0} \frac{1}{z} T(z) T(0)$ is a dimension 4 Virasoro descendant. Its "fake" torus one-point function is given by (see Appendix D for a derivation)

$$
\begin{aligned}
\langle T * T\rangle_{\tau}^{\prime}= & {\left[\left(\frac{1}{2 \pi i} \partial_{\tau}\right)^{2}-\frac{1}{6} \frac{1}{2 \pi i} \partial_{\tau}+\frac{k}{60}\right] Z_{\mathrm{Vir}}(\tau) } \\
& +2 \sum_{n=1}^{\infty} \operatorname{Tr}_{\operatorname{Vir}(k)} L_{-n} L_{n} e^{2 \pi i \tau\left(L_{0}-k\right)}
\end{aligned}
$$

By matching (4.11) with

$$
Z_{\text {saddle }}=\exp \left[k S_{0}+S_{1}+\frac{1}{k} S_{2}+\frac{1}{k^{2}} S_{3}+\cdots\right],
$$

we find precise agreement with the expression for $S_{0}, S_{1}$ as given by (2.4), (4.1) up to order $\nu^{4}$. Further, we can derive all $S_{l}$ 's from $Z_{\text {fake }}$ up to order $\nu^{4}$ as well, at least as an expansion in $q, s$. We can give the closed form expressions up to order $\nu^{2}$,

$$
\begin{aligned}
S_{1}= & -\sum_{n=2}^{\infty} \ln \left[\left(1-q^{n}\right)\left(1-s^{n}\right)\right]+(2 \pi i \nu)^{2} \\
& \times\left(\frac{2 q}{1-q} \hat{E}_{2}^{\sigma}+\frac{2 s}{1-s} \hat{E}_{2}^{\rho}-4 \hat{E}_{2}^{\rho} \hat{E}_{2}^{\sigma}\right)+\mathcal{O}\left(\nu^{4}\right), \\
S_{2}= & \frac{(2 \pi i \nu)^{2}}{12}\left(\frac{q}{1-q}-\hat{E}_{2}^{\rho}\right)\left(\frac{s}{1-s}-\hat{E}_{2}^{\sigma}\right)+\mathcal{O}\left(\nu^{4}\right), \\
S_{3}= & \mathcal{O}\left(\nu^{4}\right) .
\end{aligned}
$$

This precisely agrees with the $\mathcal{O}\left(\nu^{2}\right)$ term in (4.3).

\section{Higher genera}

Our proposals have straightforward generalizations to partition functions of higher genus $g$. The genus two modular group $\operatorname{Sp}(4, \mathbf{Z})$ will be replaced by $\mathrm{Sp}(2 g, \mathbf{Z})$, and the partition function transforms under the modular group with a certain weight. Due to the conformal anomaly, there is some ambiguity in defining the partition function. In the genus two case, it is natural to demand the partition function to have weight $2 k$; so that in the separating degeneration limit, the partition function behaves like $\epsilon^{-2 k}$. For $g>2$, requiring this kind of behavior in the factorization limit would mean 
that the partition function should have weight $(4-4 / g) k$, as explained in Appendix E, by examining the case of hyperelliptic curves. To be consistent with our genus two notation, we will continue to call this partition function $Z_{k, g}^{\bmod }(\Omega)$. A more natural and convenient choice is to simply eliminate the singularities, so that the partition function has weight $12 k$. This is the equivalent to the statement that the partition function is a holomorphic section of the $12 k$ th tensor power of the determinant line bundle $\mathcal{L}$ over the moduli space of the Riemann surface [1]. We will denote this partition function by $T_{k, g}(\Omega)$, consistent with our notation in $(2.1)$.

The extension of the definition of $Z_{\text {fake }}$ to higher genera is in principle straightforward: the genus $g$ partition function of a general CFT can be obtained by sewing sphere $2 g$-point functions, with $g$ propagators connecting pairs of operators, corresponding to the $g$ pinching handles; $Z_{\text {fake }}$ is defined by choosing the 1-cycles around each of the $g$ handles to be contractible in the handlebody filling $\Sigma_{g}$ and sewing together all sphere $2 g$-point functions of Virasoro descendants of the identity. When the Riemann surface is hyperelliptic, of the form $y^{2}=\prod_{i=1}^{2 g+2}\left(x-e_{i}\right), Z_{\text {fake }}$ can be computed explicitly from

$$
\tilde{Z}_{\text {fake }}\left(e_{1}, \ldots, e_{2 g+2}\right)=\left(\prod_{1 \leq i<j \leq 2 g+2} e_{i j}\right)^{3 k} \frac{\left\langle\prod_{s=1}^{g+1} \Psi_{e_{2 s-1,2 s}}\left(\frac{e_{2 s-1}+e_{2 s}}{2}\right)\right\rangle}{\left(\prod_{r=1}^{g+1} e_{2 r-1,2 r}\right)^{3 k}},
$$

where $e_{i j} \equiv e_{i}-e_{j}$. Note that we have chosen the exponent $3 k$ instead of $k$ in the first factor on the RHS of (5.1), anticipating the full partition function $\left(T_{k, g}(\Omega)\right)$ to be a modular form of weight $12 k$. The $e_{i}$ 's are ordered so that the branch cuts connecting $e_{2 s-1}$ and $e_{2 s}(s=1, \ldots, g)$ correspond to the $g$ handles that are filled in by the gravitational instanton. For more general Riemann surfaces, one needs to know the mapping between the pinching parameters and the periods, as well as the universal "holomorphic correction" factor, in order to calculate $Z_{\text {fake }}$ explicitly.

An obvious conjecture is that $Z_{\text {fake }}=Z_{\text {saddle }}=\exp \left(k \hat{S}_{0}+\sum_{l \geq 1} k^{1-l} S_{l}\right)$ should hold for all $k$ and all genus $g$. We used the notation $\hat{S}_{0}$ to indicate the convention that the partition function is a weight $12 k$ modular form, with no singularities. We propose an exact expression for $\hat{S}_{0}$ to be

$$
e^{\hat{S}_{0}}=\mathcal{F}(\Omega)^{12}
$$


where $\mathcal{F}(\Omega)$ is given by (3.5). Note that in our convention, the genus $g$ partition function $T_{\phi, g}(\Omega)$ of a chiral boson is simply 1. This is explained in Appendix E when the Riemann surface is a hyperelliptic curve. More generally, we can regard $T_{\phi, g}(\Omega)$ as a canonical section of the line bundle $\mathcal{L}^{1 / 2}$. Equation (5.2) is consistent with the genus one answer and our proposal in the genus two case: $e^{\hat{S}_{0}}=e^{S_{0}} \eta^{24}$ for $g=1$ and $e^{\hat{S}_{0}}=e^{S_{0}} \chi_{10}$ for $g=2$. The conjectured expression for $S_{1}$, (4.1), clearly generalizes to higher genera as well.

The formula (4.10) for the full handlebody contribution to the partition function, by summing over modular images of $Z_{\text {fake, also admits a straight- }}$ forward generalization to $g>2$,

$$
\hat{Z}_{h . b .}(k, \Omega)=\sum_{\Gamma_{\infty} \backslash \operatorname{Sp}(2 g, \mathbf{Z})} \operatorname{det}(C \Omega+D)^{-12 k} \hat{Z}_{\text {fake }}(k, \gamma \cdot \Omega)
$$

where the hat emphasizes our convention for the weight $12 k$ partition function. In particular, $Z_{\text {fake }}$ is $\Gamma_{\infty}$ invariant by the tree level factorization of the correlation functions of Virasoro descendants of the identity. A crucial new feature in the $g>2$ case is that the period matrix of a Riemann surface lies in a subspace of Siegel upper-half space of non-zero co-dimension. The genus $g$ partition function in general will not be a Siegel modular form (or quotients thereof), but a Teichmüller modular form. Nevertheless, (5.3) could still be well defined for $g>2$ and give the full handlebody contribution. Notably, modular invariance and the factorization of $Z_{\text {grav }}(k, \Omega)$ (when handles are pinched) as given by (5.3) appear to be manifest. The factorization property follows from: (a) $Z_{\text {fake }}$ is singular only when the pinching 1-cycle is contractible in the filling three-manifold; and (b) when such a 1-cycle is pinched, $Z_{\text {fake }}$ factorizes correctly by construction. If one could also fix the non-handlebody contributions to all genera, then one would be able to reconstruct all correlation functions of the dual ECFT by expanding the partition functions near various degenerating limits of the Riemann surfaces [16].

\section{Non-handlebodies}

Let us briefly describe the case when the gravitational instanton, represented by a hyperbolic three-manifold $M$ with conformal boundary $\Sigma_{g}$, is not a handlebody. In general, $M$ can be modeled as $\mathbf{H}_{3} / G$, where $G$, being the 
fundamental group of $M$, is a Kleinian group and is not a Schottky group. This would be the case whenever $G$ is not freely generated. ${ }^{7}$

A simple example of such a non-handlebody $M$ is as follows. ${ }^{8}$ First consider a quotient of $\mathbf{H}_{3}$ by a Fuchsian group (a subgroup of $\operatorname{SL}(2, \mathbf{R}) \subset$ $\mathrm{SL}(2, \mathbf{C})$ ), resulting in a hyperbolic three-manifold whose conformal boundary is two copies of $\Sigma_{g}$. We will call it $\widetilde{M}_{1}$. The metric on $\widetilde{M}_{1}$ can be written as

$$
d s^{2}=d \rho^{2}+\cosh ^{2} \rho d s_{\Sigma}^{2}
$$

where $d s_{\Sigma}^{2}$ is a hyperbolic metric on $\Sigma_{g}$. Suppose $\Sigma_{g}$ further admits a fixedpoint free orientation reversing involution. Let $\iota$ be this involution together with the $\mathbf{Z}_{2}$ symmetry $\rho \mapsto-\rho$. Then the identification of $\widetilde{M}_{1}$ by $\iota$ gives a hyperbolic three-manifold $M_{1}$, with conformal boundary $\Sigma_{g}$, which is not a handlebody. Although the manifold $M_{1}$ was constructed assuming the existence of a $\mathbf{Z}_{2}$ involution on $\Sigma_{g}$, a hyperbolic metric exists on $M_{1}$ for any complex structure on $\Sigma_{g}$. Furthermore, the conjugacy classes of the corresponding Kleinian group $G$ varies holomorphically with the complex moduli of $\Sigma_{g} \cdot{ }^{9}$

While a handlebody filling $\Sigma_{g}$ is invariant under $\Gamma_{\infty} \subset \operatorname{Sp}(2 g, \mathbf{Z}), M_{1}$ is invariant under a different subgroup $Z(\iota)$ - the commutant of $\iota$ in $\operatorname{Sp}(2 g, \mathbf{Z})$. There are other modular images of $M_{1}$, labeled by the coset $Z(\iota) \backslash \operatorname{Sp}(2 g, \mathbf{Z})$. For example suppose $g=2$, and then $\iota$ is given by the $\operatorname{Sp}(4, \mathbf{Z})$ matrix

$$
\iota=\left(\begin{array}{llll}
0 & 1 & 0 & 0 \\
1 & 0 & 0 & 0 \\
0 & 0 & 0 & 1 \\
0 & 0 & 1 & 0
\end{array}\right)
$$

The period matrix $\Omega$ should then satisfy $\Omega=-\overline{\iota \cdot \Omega}$, namely $\rho=-\bar{\sigma}, \nu \in i \mathbf{R}$.

\footnotetext{
${ }^{7}$ If $G$ is freely generated, but not purely loxodromic, it can be "approximated" as a limit of Schottky groups, as subsets of $\mathrm{SL}(2, \mathbf{C})$. The formula (3.5) then suggests that the contribution from such instantons should vanish, as some of the $q_{\gamma}$ 's approach 1 in the limit.

${ }^{8}$ Thanks to D. Gaiotto, E. Witten, C. McMullen for pointing out this example.

${ }^{9} M_{1}$ belongs to an "extreme" class of hyperbolic three-manifolds with conformal boundary $\Sigma_{g}$, in that $\pi_{1}\left(\Sigma_{g}\right)$ injects into $\pi_{1}\left(M_{1}\right)$. Most hyperbolic threemanifolds with conformal boundary $\Sigma_{g}$ do not have this property; although they may be obtained (topologically) from such manifolds with $\pi_{1}$-injective boundary by attaching 2-handles.
} 
We have not computed the regularized Einstein-Hilbert action of $M_{1}$, or any other non-handlebody instantons. It is also unclear to us how to generalize our conjectured formula for the fake CFT partition function $Z_{\text {fake }}(k, \Omega)$ to the case when $M$ is not a handlebody.

\section{Summary and questions}

We have provided non-trivial evidences for the conjectures in Section 2. Our most conservative conjecture is the statement $\ln Z_{\text {fake }}(k, \Omega)=k S_{0}+$ $S_{1}+\mathcal{O}(1 / k)$, both sides being well defined and explicitly computable as a series expansion in the pinching parameter $\epsilon$. We have checked it up to order $\epsilon^{4}$. It would be nice to prove it. It would also be nice to compute $1 / k$

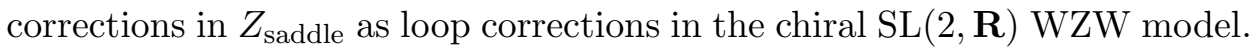

One of the most important questions is how to compute the contributions from the non-handlebody gravitational instantons to the partition function. We have seen that the contribution from handlebodies alone already captures the polar part of the expected dual ECFT partition function. This suggests that the non-handlebodies may only contribute to the non-polar part of the partition function.

On the CFT side, the most important question is whether the dual ECFTs actually exist as unitary CFTs. If we can sum up all the gravitational instanton contributions, we should obtain the full partition function of the dual ECFT. Such partition functions are modular invariant and should factorize correctly in the degenerating limits of the Riemann surface; the contribution from the handlebodies already seems to satisfy these consistency conditions by itself. According to Friedan and Shenkar [16], all correlation functions of the CFT can then be consistency recovered from these partition functions. Even if this is the case, the resulting CFT is not obviously unitary. These questions are left to future work.

\section{Acknowledgments}

I am indebted to S. Minwalla for raising the questions that motivated this work and for collaborations through a large part of it. I am grateful to D. Gaiotto, D. Jafferis, A. Strominger and E. Witten for comments on earlier drafts of the paper and to C. McMullen for explaining to me aspects of hyperbolic three-manifolds. I would like to thank C. Beasley, V. Bouchard, F. Denef, J. Maldacena, A. Maloney, G. Moore, B. Pioline, A. Simons, M. Spradlin, A. Tomasiello, A. Volovich and D. Zagier for discussions at various occasions. I also thank the hospitality of Ecole Normale Suprieure, 
LPTHE, and the organizers of XXXVIIth Paris Summer Institute on Black Holes, Black Rings and Modular Forms, where this work was initiated. I am supported by a Junior Fellowship from the Harvard Society of Fellows.

\section{Appendix A. Genus two Siegel modular forms}

\section{A.1. Some basic properties and conventions}

The genus two weight $1 / 2$ theta series are defined as a function of the period matrix $\Omega$ as

$$
\Theta[\mathbf{a}, \mathbf{b}]=\sum_{\mathbf{n} \in \mathbf{Z} \times \mathbf{Z}} \exp [\pi i(\mathbf{n}+\mathbf{a}) \cdot \Omega \cdot(\mathbf{n}+\mathbf{a})+2 \pi i(\mathbf{n}+\mathbf{a}) \cdot \mathbf{b}],
$$

where $\mathbf{a}, \mathbf{b}$ take values among the vectors $(0,0),(0,1 / 2),(1 / 2,0),(1 / 2,1 / 2)$. We will also use an alternative notation, denoting $\Theta\left[\left(1 / 2 a_{1}, 1 / 2 a_{2}\right),\left(1 / 2 b_{1}\right.\right.$, $\left.1 / 2 b_{2}\right)$ ] by $\Theta_{a_{1} a_{2} b_{1} b_{2}}$. Only 10 out of 16 expressions in (A.1) are nonvanishing. They are

$$
\begin{aligned}
& \Theta_{0000}, \quad \Theta_{0001}, \quad \Theta_{0010}, \quad \Theta_{0011}, \quad \Theta_{0100}, \quad \Theta_{0110}, \quad \Theta_{1000}, \quad \Theta_{1001}, \\
& \Theta_{1100}, \Theta_{1111} \text {. }
\end{aligned}
$$

A set of generators of the ring of entire Siegel modular forms (according to the convention of [5]) are

$$
\begin{aligned}
\tilde{\psi}_{4} & =\frac{1}{4} \sum_{\mathbf{a}, \mathbf{b}} \Theta[\mathbf{a}, \mathbf{b}]^{8}, \\
\Delta_{10} & =\frac{1}{2^{12}} \prod_{\mathbf{a}, \mathbf{b}} \Theta[\mathbf{a}, \mathbf{b}]^{2}, \\
F_{12} & =\frac{1}{4} \sum_{\mathbf{a}, \mathbf{b}} \Theta[\mathbf{a}, \mathbf{b}]^{24}, \\
\tilde{\psi}_{6} & =P_{0}^{3}-9 P_{0}\left(P_{1}^{2}+P_{2}^{2}+P_{3}^{2}-4 P_{4}^{2}\right)+54 P_{1} P_{2} P_{3},
\end{aligned}
$$

where the $P_{i}$ are related to the $\Theta$ 's by

$$
\begin{aligned}
& \Theta_{0000}^{4}=P_{0}+P_{1}+P_{2}+P_{3}, \\
& \Theta_{0001}^{4}=P_{0}-P_{1}+P_{2}-P_{3}, \\
& \Theta_{0010}^{4}=P_{0}+P_{1}-P_{2}-P_{3}, \\
& \Theta_{0011}^{4}=P_{0}-P_{1}-P_{2}+P_{3}, \\
& \Theta_{0100}^{4}=2 P_{1}+2 P_{4},
\end{aligned}
$$




$$
\begin{aligned}
& \Theta_{0110}^{4}=2 P_{1}-2 P_{4}, \\
& \Theta_{1000}^{4}=2 P_{2}+2 P_{4}, \\
& \Theta_{1001}^{4}=2 P_{2}-2 P_{4}, \\
& \Theta_{1100}^{4}=2 P_{3}+2 P_{4}, \\
& \Theta_{1111}^{4}=2 P_{3}-2 P_{4} .
\end{aligned}
$$

Note that there are five linear relations among the $10 \Theta^{4}$ 's, so that (A.4) consistently determine $P_{0,1,2,3,4}$. The generators $\psi_{4}, \psi_{6}, \chi_{10}, \chi_{12}$ of [3] are related to those in (A.3) by

$$
\begin{aligned}
\psi_{4} & =\frac{1}{4} \tilde{\psi}_{4}, \\
\psi_{6} & =\frac{1}{16} \tilde{\psi}_{6}, \\
\chi_{10} & =4 \Delta_{10}, \\
\chi_{12} & =\frac{96}{2^{13} 3^{4}}\left(\frac{9}{11} F_{12}-\tilde{\psi}_{4}^{3}+\frac{2}{11} \tilde{\psi}_{6}^{2}\right) .
\end{aligned}
$$

\section{A.2. Averaging over the modular group}

To begin let us recall the case of $\operatorname{SL}(2, \mathbf{Z})$ modular forms. See [17] for example. Given a $\Gamma_{\infty}$-invariant function $h(\tau)$, its Poincaré series of weight $w$ is defined by the summation over $\mathrm{SL}(2, \mathbf{Z})$ images,

$$
\mathbf{P}_{w} h(\tau)=\sum_{\gamma \in \Gamma_{\infty} \backslash \mathrm{SL}(2, \mathbf{Z})}(c \tau+d)^{-w} h\left(\frac{a \tau+b}{c \tau+d}\right)
$$

For $w \geq 4$, it is well known that $\mathbf{P}_{w}\left(q^{n}\right)(n>0)$ span the space of weight $w$ cusp forms. In particular, they vanish when $w=4,6,8,10,14$. For these values of $w$, a weight $w$ weakly holomorphic modular form $f_{w}(\tau)=\sum a_{n} q^{n}$ is given by the Poincare series of its polar (and constant) part,

$$
f_{w}^{-}(\tau)=\sum_{n \leq 0} a_{n} q^{n}
$$

Namely, $f_{w}(\tau)=\mathbf{P}_{w} f_{w}^{-}(\tau)$. The situation is more complicated for higher weights. When there are weight $w$ cusp forms, $\mathbf{P}_{w} q^{n}$ can be non-zero for arbitrarily large $n$. They can be determined in terms of the cusp forms as 
follows. Define the inner product

$$
\langle f, g\rangle_{w}=\int_{\mathcal{F}} f(\tau) \overline{g(\tau)} \tau_{2}^{w-2} d^{2} \tau
$$

where $\mathcal{F}$ is the fundamental domain for $\operatorname{SL}(2, \mathbf{Z})$. If $\chi(\tau)=\sum_{n>0} b_{n} q^{n}$ is a cusp form, then

$$
\left\langle\chi, \mathbf{P}_{w} q^{n}\right\rangle_{w}=(w-2) !(4 \pi n)^{1-w} b_{n}
$$

Let $\chi_{i}$ be a basis of weight $w$ cusp forms, with $\left\langle\chi_{i}, \chi_{j}\right\rangle_{w}=c_{i j}$, then we have (for $n>0$ )

$$
\mathbf{P}_{w} q^{n}=\sum_{i, j}\left\langle\chi_{i}, \mathbf{P}_{w} q^{n}\right\rangle_{w}\left(c^{-1}\right)^{i j} \chi_{j}(\tau)
$$

Things are simple if we only want to compare the polar terms. For positive weight $w$, if $h(\tau)$ is a $\Gamma_{\infty}$-invariant function, which is regular away from $\tau=i \infty$, and if $f_{w}(\tau)=\mathbf{P}_{w} h(\tau)$, then $f_{w}^{-}(\tau)=h^{-}(\tau)$. We can also relax the positivity condition on $w$, while assuming that $\mathbf{P}_{w} h$ converges absolutely and is regular away from $\tau=i \infty$, then $\mathbf{P}_{w} h(\tau)=h(\tau)+\mathcal{O}\left(q^{0}\right)$.

Now let us turn to $\operatorname{Sp}(4, \mathbf{Z})$ Siegel modular forms. Given a $\Gamma_{\infty}$-invariant function $h(\Omega)$, its $\operatorname{Sp}(4, \mathbf{Z})$ Poincaré series of weight $w$ is defined as

$$
\mathbf{P}_{w} h(\Omega)=\sum_{\gamma \in \Gamma_{\infty} \backslash \operatorname{Sp}(4, \mathbf{Z})}(\operatorname{det}(C \Omega+D))^{-w} h(\gamma \cdot \Omega)
$$

The Fourier basis is

$$
e_{T}(\Omega)=e^{2 \pi i \operatorname{Tr}(T \Omega)}
$$

where $T$ is a symmetric $2 \times 2$ matrix with integer entries. $e_{T}$ is invariant under $\Gamma_{\infty}^{\prime} \subset \operatorname{Sp}(4, \mathbf{Z})$, consisting of elements of the form

$$
\left(\begin{array}{cc} 
\pm 1 & B \\
0 & \pm 1
\end{array}\right)
$$

The sum

$$
h_{T}(\Omega)=\sum_{\gamma \in \Gamma_{\infty}^{\prime} \backslash \Gamma_{\infty}} e_{T}(\gamma \cdot \Omega)=\sum_{A \in P \operatorname{SL}(2, \mathbf{Z})} e_{A^{\mathrm{T}} T A}(\Omega)
$$

converges for positive definite $T$, with $\Omega$ taking values on the Siegel upper half space. These are the analogs of the $q^{n}$ 's $(n>0)$ in the $\operatorname{SL}(2, \mathbf{Z})$ case. 
For a pair of cusp forms $f(\Omega), g(\Omega)$ of weight $w$, one can define their inner product

$$
\langle f, g\rangle_{w}=\int_{\mathcal{F}} f_{w}(\Omega) \overline{g(\Omega)}(\operatorname{det} \operatorname{Im} \Omega)^{w-3} d \Omega d \bar{\Omega}
$$

where we wrote $d \Omega \equiv d \rho d \nu d \sigma$, and $\mathcal{F}$ for the fundamental domain of $\operatorname{Sp}(4, \mathbf{Z})$ on the Siegel upper half space $\mathbf{H}$. If $f(\Omega)=\sum_{T>0} a_{T} e_{T}(\Omega)$ is a cusp form, then

$$
\left\langle f, \mathbf{P}_{w} h_{T}\right\rangle_{w}=a_{T} \int_{M_{2 \times 2} \geq 0} e^{-2 \pi T r(T M)}(\operatorname{det} M)^{w-3} d M .
$$

We see that once again, $\mathbf{P}_{w} h_{T}$ are generically non-vanishing $(T>0)$ and can be expressed as a linear combination of basis cusp forms using (A.16).

In practice, computing $\mathbf{P}_{w} h(\Omega)$ based on (A.16) is not easy. Things simplify if we only look at the polar terms. One should be cautious that the moduli space of a genus two Riemann surface is not the quotient of $\mathbf{H}$ by $\operatorname{Sp}(4, \mathbf{Z})$, but rather the quotient of $\mathbf{H}-\mathcal{D}$, where $\mathcal{D}$ is the set of $\operatorname{Sp}(4, \mathbf{Z})$ images of $\nu=0$. We are interested in Siegel modular forms of the form $f_{w}(\Omega)=T(\Omega) \chi_{10}(\Omega)^{-k}$, where $T(\Omega)$ is a (weight $w+10 k$ ) entire modular form. Near $q=s=\nu=0$, we have $f_{w}(\Omega)=\mathcal{O}\left(q^{-k} s^{-k} \nu^{-2 k}\right)$. A useful fact is the following simple lemma.

Lemma A.1. If $w<10, f_{w}$ is a weight $w \operatorname{Sp}(4, \mathbf{Z})$ modular forms possibly with poles along $\mathcal{D}$, and if $f_{w}(\Omega)=\mathcal{O}(q, s)$, then $f_{w}(\Omega)=0$.

To see this, suppose $f_{w}=T \chi_{10}^{-k}$, and $T=P\left(\psi_{4}, \psi_{6}, \chi_{12}\right)+\chi_{10} Q\left(\psi_{4}, \ldots\right)$, where $P$ is a non-zero polynomial in $\psi_{4}, \psi_{6}, \chi_{12}$. Restricting to $\nu=0$, we have $P\left(E_{4}(\rho) E_{4}(\sigma), E_{6}(\rho) E_{6}(\sigma), \Delta_{12}(\rho) \Delta_{12}(\sigma)\right)=\mathcal{O}\left(q^{k+1}, s^{k+1}\right)$. This is only possible if $P=\Delta_{12}(\rho)^{k+1}(\cdots)+\Delta_{12}(\sigma)^{k+1}(\cdots)$, which would require $w+10 k \geq 12(k+1)$, contradicting the assumption $w<10$.

Generally, suppose $h(\Omega)$ is a $\Gamma_{\infty}$-invariant holomorphic function, possibly with finite order poles along $\rho=i \infty, \sigma=i \infty$ or $\nu=0$, as well as their $\Gamma_{\infty}$ images, but it is otherwise regular everywhere on $\overline{\mathbf{H}}$. Suppose that the Poincaré series $\mathbf{P}_{w} h(\Omega)$ converges absolutely. Then we have

Lemma A.2. $\mathbf{P}_{w} h(\Omega)=h(\Omega)+\mathcal{O}\left(q^{0}, s^{0}\right)$. 
We need to show that for $\gamma \in \operatorname{Sp}(4, \mathbf{Z})-\Gamma_{\infty}, h(\gamma \cdot \Omega)=\mathcal{O}\left(q^{0}\right)+\mathcal{O}\left(s^{0}\right)$. Suppose $\mathbf{P}_{w} h(\Omega)=T \chi_{10}^{-k}$ for some entire Siegel modular form $T$. Writing

$$
\gamma=\left(\begin{array}{ll}
A & B \\
C & D
\end{array}\right), \quad C \neq 0
$$

we can consider two cases: (a) $\operatorname{det} C \neq 0$. In this case, $\gamma \cdot \Omega=A C^{-1}+$ $\mathcal{O}(\nu / \rho \sigma)$ as $\rho, \sigma \rightarrow i \infty$, and we have

$$
|h(\gamma \cdot \Omega)|<N_{\gamma}\left|\frac{\operatorname{det}(C \Omega+D)}{\nu}\right|^{2 k}
$$

for small $\nu$. (b) $\operatorname{rk} C=1$. In this case, we have

$$
\gamma \cdot \Omega \sim \frac{\rho \sigma A C^{*}}{\operatorname{det}(C \Omega+D)},
$$

where $C^{*}$ stands for the cofactor matrix of $C$. Then, we can bound

$$
|h(\gamma \cdot \Omega)|<N_{\gamma}\left|e^{-2 \pi i k\left\|A C^{*}\right\| \frac{\rho \sigma}{\operatorname{det}(C \Omega+D)}}\right|\left|\frac{\operatorname{det}(C \Omega+D)}{\nu}\right|^{2 k}
$$

for small $\nu$. In either case, the image of $h(\Omega)$ under $\gamma$ in the Poincaré series can only contribute to terms of order $\mathcal{O}\left(q^{0}, s^{0}\right)$ and never to terms that polar in both $q$ and $s$, hence proving the lemma.

In other words, for a function $f(\Omega)=\sum a_{n, m, r} q^{n} s^{m} \sin (\pi \nu)^{2 r}$, if we define its polar part as

$$
f^{-}(\Omega)=\sum_{n, m<0, r \in \mathbf{Z}} a_{n, m, r} q^{n} s^{m} \sin (\pi \nu)^{2 r},
$$

then $\left(\mathbf{P}_{w} h\right)^{-}=h^{-}$for $h(\Omega)$ satisfying the regularity condition above. Note that despite the summation in (A.17) is over all $r, a_{n, m, r}$ is only nonvanishing for a finite set of values of $r$ for the type of modular forms we are considering. One should also be cautious that our definition of polar part is not $\Gamma_{\infty}$ invariant.

In our application, the handlebody contribution to the partition function $Z_{h . b .}(k, \Omega)$ is given by the Poincaré series of $Z_{\text {fake }}(k, \Omega)$, and if we assume 
that $Z_{\text {fake }}$ satisfy the regularity criteria of $h(\Omega)$ above, ${ }^{10}$ then the polar terms of $Z_{h . b .}(\Omega)$ and $Z_{\text {fake }}(k, \Omega)$ in $q, s$ must agree. On the other hand, the polar terms in $q, s$ of $Z_{k}^{\bmod }$ do not involve the contribution from three-point functions of non-trivial primaries, and hence necessarily agrees with $Z_{\text {fake }}$ (if a consistent ECFT partition function exists). Therefore, we conclude that the handlebody contribution already gives all the correct polar terms of $Z_{k}^{\text {mod }}$. In fact, the terms of order $q^{0} s^{n \leq 0}$ and $q^{n \leq 0} s^{0}$ in $Z_{\text {fake }}$ also agree with $Z_{k}^{\text {mod }}$, as they do not involve non-trivial primaries either. One might then expect $Z_{h . b .}=\mathbf{P}_{2 k} Z_{\text {fake }}$ to capture these terms as well. This does not follow from our lemma, although there could be better estimates on $\mathbf{P}_{w} h(\Omega)-h(\Omega)$.

\section{Appendix B. The Schottky parameterization}

\section{B.1. Generalities}

In this subsection, we describe some useful properties of the Schottky parameterization of a Riemann surface. The Schottky group $\Gamma$ is a subgroup of $\operatorname{SL}(2, \mathbf{C})$ freely generated by $g$ elements $\gamma_{1}, \ldots \gamma_{g}$, which acts on $\mathbf{P}^{1}$ by Mobius transformation. It is convenient to parameterize a group element $\gamma$ by its fixed points $\xi, \eta$ and its multiplier $q_{\gamma}$,

$$
\frac{\gamma(z)-\eta}{\gamma(z)-\xi}=q_{\gamma} \frac{z-\eta}{z-\xi}
$$

Geometrically, $\gamma$ maps a circle $C$ around $\xi$ to another circle $C^{\prime}$ around $\eta$; it maps the domain outside of $C$ (or $C^{\prime}$ ) to the disc bounded by $C^{\prime}$ (or $C$ ).

A Riemann surface $\Sigma_{g}$ of a given complex structure can be realized as the quotient of $\mathbf{P}^{1}$ (excluding a suitable zero measure set) by $\Gamma$. Specializing to the genus two case, we will choose a pair of generators of $\Gamma, \alpha$ and $\beta$,

$$
\frac{\alpha(z)-\eta_{1}}{\alpha(z)-\xi_{1}}=q_{\alpha} \frac{z-\eta_{1}}{z-\xi_{1}}, \quad \frac{\beta(z)-\eta_{2}}{\beta(z)-\xi_{2}}=q_{\beta} \frac{z-\eta_{2}}{z-\xi_{2}} .
$$

The Schottky space is parameterized by $q_{\alpha}, q_{\beta}, \xi_{1}, \xi_{2}, \eta_{1}, \eta_{2}$, up to the $\mathrm{SL}(2, \mathbf{C})$ action by conjugation. The relation between the Schottky parameters and the period matrix elements $\rho, \sigma, \nu$ is given for example in [12], by

\footnotetext{
${ }^{10}$ This is not quite the case in general: the genus one answer, $Z_{\text {fake }}^{g=1}=$ $q^{-k} \prod_{n=2}^{\infty}\left(1-q^{n}\right)^{-1}=q^{-k+1 / 24}(1-q) \eta(\tau)^{-1}$, diverges at the $\operatorname{SL}(2, \mathbf{Z})$ images of $\tau=i \infty$ as well. Nevertheless, this problem can be fixed if we multiply $Z_{\text {fake }}^{g=1}$ by $\eta(\tau)$, while raising the weight of the Poincaré series by $1 / 2$. In the genus two case, it is likely that multiplying $Z_{\text {fake }}$ by $\chi_{10}^{1 / 24}$ will suffice.
} 
the following formulae:

$$
\begin{aligned}
e^{2 \pi i \rho} & =q_{\alpha} \prod_{\gamma=\beta^{n} \ldots \beta^{m}}\left(\frac{\gamma\left(\eta_{1}\right)-\eta_{1}}{\gamma\left(\xi_{1}\right)-\eta_{1}} \frac{\gamma\left(\xi_{1}\right)-\xi_{1}}{\gamma\left(\eta_{1}\right)-\xi_{1}}\right), \\
e^{2 \pi i \sigma} & =q_{\beta} \prod_{\gamma=\alpha^{n} \cdots \alpha^{m}}\left(\frac{\gamma\left(\eta_{2}\right)-\eta_{2}}{\gamma\left(\xi_{2}\right)-\eta_{2}} \frac{\gamma\left(\xi_{2}\right)-\xi_{2}}{\gamma\left(\eta_{2}\right)-\xi_{2}}\right), \\
e^{2 \pi i \nu} & =\frac{\eta_{12} \xi_{12}}{\left(\xi_{1}-\eta_{2}\right)\left(\eta_{1}-\xi_{2}\right)} \prod_{\gamma=\alpha^{n} \cdots \beta^{m}}\left(\frac{\gamma\left(\eta_{1}\right)-\eta_{2}}{\gamma\left(\xi_{1}\right)-\eta_{2}} \frac{\gamma\left(\xi_{1}\right)-\xi_{2}}{\gamma\left(\eta_{1}\right)-\xi_{2}}\right) .
\end{aligned}
$$

The product in the first line runs through all distinct elements $\gamma$ corresponding to a word with $\beta$ or $\beta^{-1}$ on the left and right ends. The product in the second line is over elements with $\alpha^{ \pm 1}$ on the left and right ends. The product in the last line is over elements whose word starts with $\alpha^{ \pm 1}$ and ends with $\beta^{ \pm 1}$.

The Schottky parameterization is $\Gamma_{\infty}$ invariant. $\Gamma_{\infty}$ as a subgroup of $\operatorname{Sp}(4, \mathbf{Z})$ is generated by integral shifts of the matrix elements of $\Omega$, as well as the $\mathrm{SL}(2, \mathbf{Z})$ transformations that acts on $\Omega$ as $\Omega \mapsto A \Omega A^{\mathrm{T}}$. The invariance under integral shifts of $\Omega$ is clear from (B.3). It is also clear that the $\operatorname{SL}(2, \mathbf{Z})$ transformation exchanging $\rho$ with $\sigma$ corresponds to swapping the generators $\alpha, \beta$ in $\Gamma$. On the other hand, the $\operatorname{SL}(2, \mathbf{Z})$ transformation sending $\rho \mapsto$ $\rho+\sigma, \sigma \mapsto \sigma$, corresponds to redefining the two generators of $\Gamma$ to be $\alpha$ and $\alpha \beta$. In particular, it follows that $\mathcal{F}(\Omega)(3.5)$, as well as out conjectured formulae for $S_{0}$ and $S_{1}$, is invariant under $\Gamma_{\infty}$.

\section{B.2. Schottky parameters in terms of periods up to $\mathcal{O}\left(\nu^{2}\right)$}

The relation between the Schottky parameters and the periods (B.3) is rather complicated. It is useful to expand it explicit in $\nu$, in the $\nu \rightarrow 0$ limit (separating degeneration). In terms of the Schottky parameters, this limits corresponds to separating $\xi_{1}, \eta_{1}$ and $\xi_{2}, \eta_{2}$ at a large distance $L ; \nu$ scales like $1 / L^{2}$.

To leading non-trivial order, only the product over elements $\beta^{n}(n \neq 0)$ contribute in the first line of (B.3). In fact, straightforward calculation shows that

$$
\frac{\beta^{n}\left(\eta_{1}\right)-\eta_{1}}{\beta^{n}\left(\xi_{1}\right)-\eta_{1}} \frac{\beta^{n}\left(\xi_{1}\right)-\xi_{1}}{\beta^{n}\left(\eta_{1}\right)-\xi_{1}}=1+\frac{\left(\eta_{1}-\xi_{1}\right)^{2}\left(\eta_{2}-\xi_{2}\right)^{2}}{L^{4}\left(q_{\beta}^{\frac{n}{2}}-q_{\beta}^{-\frac{n}{2}}\right)^{2}}+\mathcal{O}\left(\frac{1}{L^{5}}\right)
$$


whereas for Schottky elements of the form $\gamma=\beta \cdots \alpha \cdots \beta$,

$$
\frac{\gamma\left(\eta_{1}\right)-\eta_{1}}{\gamma\left(\xi_{1}\right)-\eta_{1}} \frac{\gamma\left(\xi_{1}\right)-\xi_{1}}{\gamma\left(\eta_{1}\right)-\xi_{1}}=1+\mathcal{O}\left(\frac{1}{L^{8}}\right) .
$$

Plugging this into the first line of (B.3), we find

$$
\begin{aligned}
e^{2 \pi i \rho} & =q_{\alpha}\left[1+\frac{\left(\eta_{1}-\xi_{1}\right)^{2}\left(\eta_{2}-\xi_{2}\right)^{2}}{L^{4}} \sum_{n \neq 0}\left(q_{\beta}^{\frac{n}{2}}-q_{\beta}^{-\frac{n}{2}}\right)^{-2}+\mathcal{O}\left(\frac{1}{L^{5}}\right)\right] \\
& =q_{\alpha}\left[1+\frac{\left(\eta_{1}-\xi_{1}\right)^{2}\left(\eta_{2}-\xi_{2}\right)^{2}}{L^{4}} \frac{1-E_{2}\left(\tau_{2}\right)}{12}+\mathcal{O}\left(\frac{1}{L^{5}}\right)\right]
\end{aligned}
$$

and similarly for $e^{2 \pi i \sigma}$. Here, $E_{2}(\tau)$ is the second Eisenstein series. For brevity, we will often use to express the Einstein series in terms of $\hat{E}_{n}^{\tau}$, defined below (3.13). In particular, $E_{2}(\tau)=1-24 \hat{E}_{2}^{\tau}, E_{4}(\tau)=1+240 \hat{E}_{4}^{\tau}$.

To calculate $\nu$, the first factor on the RHS of the third line of (B.3) gives the dominant contribution,

$$
2 \pi i \nu=\frac{\left(\eta_{1}-\xi_{1}\right)\left(\eta_{2}-\xi_{2}\right)}{L^{2}}+\mathcal{O}\left(\frac{1}{L^{3}}\right) .
$$

The next order corrections come from the product over $\gamma$ of the form $\alpha^{n} \cdots \beta^{m}(n, m \neq 0)$, of

$$
\frac{\gamma\left(\eta_{1}\right)-\eta_{2}}{\gamma\left(\xi_{1}\right)-\eta_{2}} \frac{\gamma\left(\xi_{1}\right)-\xi_{2}}{\gamma\left(\eta_{1}\right)-\xi_{2}}=1+\mathcal{O}\left(\frac{1}{L^{6}}\right),
$$

(B.6) and (B.7) express $\rho, \sigma, \nu$ in terms of the Schottky parameters to order $\nu^{2}$.

\section{B.3. $\mathcal{O}\left(\nu^{4}\right)$}

In this subsection, we will carry out the computation in the previous section to the next order $\nu^{4}$. One must expand $\nu$ in terms of the Schottky parameters to order $1 / L^{6}$. We will omit the explicit expression as it is too lengthy. Inverting it, we can express $1 / L^{2}$ in terms of $\nu$ and express everything as an expansion in powers of $\nu$. Define

$$
A_{\gamma} \equiv \frac{\gamma\left(\eta_{1}\right)-\eta_{1}}{\gamma\left(\xi_{1}\right)-\eta_{1}} \frac{\gamma\left(\xi_{1}\right)-\xi_{1}}{\gamma\left(\eta_{1}\right)-\xi_{1}}
$$


as in the product in the first line of (B.3). After tedious but straightforward calculations, we find

$$
\begin{aligned}
A_{\beta^{n}} & =1+\frac{(2 \pi i \nu)^{2}+\left(\frac{1}{12}-8 \hat{E}_{2}^{\alpha} \hat{E}_{2}^{\beta}\right)(2 \pi i \nu)^{4}}{\left(q_{\beta}^{\frac{n}{2}}-q_{\beta}^{-n / 2}\right)^{2}}+\frac{(2 \pi i \nu)^{4}}{\left(q_{\beta}^{n / 2}-q_{\beta}^{-n / 2}\right)^{4}}+\mathcal{O}\left(\nu^{6}\right) . \\
A_{\alpha^{n} \beta^{m}} & =1+\frac{(2 \pi i \nu)^{3}}{\left(q_{\alpha}^{n / 2}-q_{\alpha}^{-n / 2}\right)^{2}\left(q_{\beta}^{m / 2}-q_{\beta}^{-m / 2}\right)^{2}}+\mathcal{O}\left(\nu^{4}\right) \\
A_{\beta^{n} \alpha^{m} \beta^{r}} & =1+\frac{(2 \pi i \nu)^{4}}{\left(q_{\beta}^{n / 2}-q_{\beta}^{-n / 2}\right)^{2}\left(q_{\alpha}^{m / 2}-q_{\alpha}^{-m / 2}\right)^{2}\left(q_{\beta}^{r / 2}-q_{\beta}^{-r / 2}\right)^{2}}+\mathcal{O}\left(\nu^{6}\right),
\end{aligned}
$$

where $q_{\alpha} \equiv e^{2 \pi i \tau_{1}}, q_{\beta} \equiv e^{2 \pi i \tau_{2}}$ and $\hat{E}_{n}^{\alpha} \equiv \hat{E}_{n}\left(\tau_{1}\right), \hat{E}_{n}^{\beta} \equiv \hat{E}_{n}\left(\tau_{2}\right)$. Furthermore, we have

$$
\begin{aligned}
\prod_{n \neq 0} A_{\beta^{n}}= & 1+(2 \pi i \nu)^{2} 2 \hat{E}_{2}^{\beta}+(2 \pi i \nu)^{4} \\
\times & {\left[-16 \hat{E}_{2}^{\alpha}\left(\hat{E}_{2}^{\beta}\right)^{2}+2\left(\hat{E}_{2}^{\beta}\right)^{2}+\frac{1}{6} E_{4}^{\beta}\right]+\mathcal{O}\left(\nu^{6}\right), } \\
\prod_{n, m, r \neq 0} A_{\beta^{n} \alpha^{m} \beta^{r}}= & 1+(2 \pi i \nu)^{4} 8 \hat{E}_{2}^{\alpha}\left(\hat{E}_{2}^{\beta}\right)^{2}+\mathcal{O}\left(\nu^{6}\right) .
\end{aligned}
$$

The periods $\rho, \sigma$ are then related by

$$
\begin{aligned}
e^{2 \pi i \rho}= & q_{\alpha} \prod_{n \neq 0} A_{\beta^{n}} \prod_{n, m, r \neq 0} A_{\beta^{n} \alpha^{m} \beta^{r}} \cdot\left(1+\mathcal{O}\left(\nu^{6}\right)\right) \\
= & q_{\alpha}\left\{1+(2 \pi i \nu)^{2} 2 \hat{E}_{2}^{\beta}+(2 \pi i \nu)^{4}\right. \\
& \left.\times\left[2\left(\hat{E}_{2}^{\beta}\right)^{2}-8 \hat{E}_{2}^{\alpha}\left(\hat{E}_{2}^{\beta}\right)^{2}+\frac{1}{6} \hat{E}_{4}^{\beta}\right]+\mathcal{O}\left(\nu^{6}\right)\right\} \\
e^{2 \pi i \sigma}= & q_{\beta}\left\{1+(2 \pi i \nu)^{2} 2 \hat{E}_{2}^{\alpha}+(2 \pi i \nu)^{4}\right. \\
& \left.\times\left[2\left(\hat{E}_{2}^{\alpha}\right)^{2}-8 \hat{E}_{2}^{\beta}\left(\hat{E}_{2}^{\alpha}\right)^{2}+\frac{1}{6} \hat{E}_{4}^{\alpha}\right]+\mathcal{O}\left(\nu^{6}\right)\right\}
\end{aligned}
$$

Using the identity

$$
\frac{1}{2 \pi i} \partial_{\tau} E_{2}(\tau)=\frac{E_{2}(\tau)^{2}-E_{4}(\tau)}{12},
$$


we can express

$$
\hat{E}_{2}^{\beta}=\hat{E}_{2}^{\sigma}+(2 \pi i \nu)^{2} \hat{E}_{2}^{\rho} \frac{-\hat{E}_{2}^{\sigma}+12\left(\hat{E}_{2}^{\sigma}\right)^{2}-5 \hat{E}_{4}^{\sigma}}{3}+\mathcal{O}\left(\nu^{4}\right)
$$

and similarly $\hat{E}_{2}^{\alpha}$ in terms of $\hat{E}_{2}^{\rho, \sigma}$. We can then invert (B.12) and express $q_{\alpha}, q_{\beta}$ in terms of the periods,

$$
\begin{aligned}
q_{\alpha}= & e^{2 \pi i \rho}\left\{1-(2 \pi i \nu)^{2} 2 \hat{E}_{2}^{\sigma}+(2 \pi i \nu)^{4}\left[2\left(\hat{E}_{2}^{\sigma}\right)^{2}+\frac{2}{3} \hat{E}_{2}^{\rho} \hat{E}_{2}^{\sigma}\right.\right. \\
& \left.\left.-\frac{1}{6} \hat{E}_{4}^{\sigma}+\frac{10}{3} \hat{E}_{2}^{\rho} \hat{E}_{4}^{\sigma}\right]+\mathcal{O}\left(\nu^{6}\right)\right\} \\
q_{\beta}= & e^{2 \pi i \sigma}\left\{1-(2 \pi i \nu)^{2} 2 \hat{E}_{2}^{\rho}+(2 \pi i \nu)^{4}\left[2\left(\hat{E}_{2}^{\rho}\right)^{2}+\frac{2}{3} \hat{E}_{2}^{\sigma} \hat{E}_{2}^{\rho}\right.\right. \\
& \left.\left.-\frac{1}{6} \hat{E}_{4}^{\rho}+\frac{10}{3} \hat{E}_{2}^{\sigma} \hat{E}_{4}^{\rho}\right]+\mathcal{O}\left(\nu^{6}\right)\right\}
\end{aligned}
$$

\section{Appendix C. Expanding $\mathcal{F}(\Omega)$ to $\mathcal{O}\left(\nu^{4}\right)$}

To determine $\mathcal{F}(\Omega)$ to order $\nu^{4}$, we must compute $q_{\alpha^{n} \beta^{m}}$ as well as $q_{\alpha^{n_{1}} \beta^{m_{1}} \alpha^{n_{2}} \beta^{m_{2}}}$. After some messy algebra, these can be expressed straightforwardly in terms of $q_{\alpha}, q_{\beta}$, expanded in powers of $1 / L$. Translating $1 / L^{2}$ to $\nu$, we find

$$
\text { (C.1) } \begin{aligned}
q_{\alpha^{n} \beta^{m}}= & \frac{(2 \pi i \nu)^{2}}{\left(q_{\alpha}^{n / 2}-q_{\alpha}^{-n / 2}\right)^{2}\left(q_{\beta}^{n / 2}-q_{\beta}^{-m / 2}\right)^{2}} \\
& +\frac{(2 \pi i \nu)^{3}\left(q_{\alpha}^{n / 2}+q_{\alpha}^{-n / 2}\right)\left(q_{\beta}^{m / 2}+q_{\beta}^{-m / 2}\right)}{\left(q_{\alpha}^{n / 2}-q_{\alpha}^{-n / 2}\right)^{3}\left(q_{\beta}^{m / 2}-q_{\beta}^{-m / 2}\right)^{3}} \\
& +(2 \pi i \nu)^{4}\left[\left(\frac{7}{12}-8 \hat{E}_{2}^{\alpha} \hat{E}_{2}^{\beta}\right)\left(q_{\alpha}^{n / 2}-q_{\alpha}^{-n / 2}\right)^{-2}\right. \\
& \times\left(q_{\beta}^{m / 2}-q_{\beta}^{-m / 2}\right)^{-2}+3\left(q_{\alpha}^{n / 2}-q_{\alpha}^{-n / 2}\right)^{-4}\left(q_{\beta}^{m / 2}-q_{\beta}^{-m / 2}\right)^{-2} \\
& +3\left(q_{\alpha}^{n / 2}-q_{\alpha}^{-n / 2}\right)^{-2}\left(q_{\beta}^{m / 2}-q_{\beta}^{-m / 2}\right)^{-4}+14\left(q_{\alpha}^{n / 2}-q_{\alpha}^{-n / 2}\right)^{-4} \\
& \left.\times\left(q_{\beta}^{m / 2}-q_{\beta}^{-m / 2}\right)^{-4}\right]+\mathcal{O}\left(\nu^{6}\right),
\end{aligned}
$$




$$
\begin{aligned}
& q_{\alpha^{n_{1} \beta^{m_{1}}} \alpha^{n_{2} \beta^{m_{2}}}}=\frac{(2 \pi i \nu)^{4}}{\left(q_{\alpha}^{n_{1} / 2}-q_{\alpha}^{-n_{1} / 2}\right)^{2}\left(q_{\alpha}^{n_{2} / 2}-q_{\alpha}^{-n_{2} / 2}\right)^{2}}+\mathcal{O}\left(\nu^{6}\right) . \\
& \left(q_{\beta}^{m_{1} / 2}-q_{\beta}^{-m_{1} / 2}\right)^{2}\left(q_{\beta}^{m_{2} / 2}-q_{\beta}^{-m_{2} / 2}\right)^{2}
\end{aligned}
$$

Taking their products, we have

$$
\begin{aligned}
\prod_{n_{1}, n_{2}, m_{1}, m_{2} \neq 0}\left(1-q_{\left.\alpha^{n_{1}} \beta^{m_{1}} \alpha^{n_{2} \beta^{m_{2}}}\right)=}\right. & 1-(2 \pi i \nu)^{4} 16\left(\hat{E}_{2}^{\rho}\right)^{2}\left(\hat{E}_{2}^{\sigma}\right)^{2}+\mathcal{O}\left(\nu^{6}\right), \\
\prod_{n, m \neq 0}\left(1-q_{\alpha^{n} \beta^{m}}^{2}\right)= & 1-(2 \pi i \nu)^{4} \frac{\left(\hat{E}_{4}^{\rho}-\hat{E}_{2}^{\rho}\right)\left(\hat{E}_{4}^{\sigma}-\hat{E}_{2}^{\sigma}\right)}{9}+\mathcal{O}\left(\nu^{6}\right), \\
\prod_{n, m \neq 0}\left(1-q_{\alpha^{n} \beta^{m}}\right)= & 1-(2 \pi i \nu)^{2} 4 \hat{E}_{2}^{\rho} \hat{E}_{2}^{\sigma}+\frac{(2 \pi i \nu)^{4}}{18} \\
& \times\left[\hat{E}_{2}^{\rho} \hat{E}_{2}^{\sigma}+24\left(\hat{E}_{2}^{\rho}\right)^{2} \hat{E}_{2}^{\sigma}+24\left(\hat{E}_{2}^{\sigma}\right)^{2} \hat{E}_{2}^{\rho}\right. \\
& +144\left(\hat{E}_{2}^{\rho}\right)^{2}\left(\hat{E}_{2}^{\sigma}\right)^{2}-7 \hat{E}_{4}^{\rho} \hat{E}_{2}^{\sigma}-7 \hat{E}_{4}^{\sigma} \hat{E}_{2}^{\rho} \\
& \left.+120 \hat{E}_{4}^{\rho}\left(\hat{E}_{2}^{\sigma}\right)^{2}+120 \hat{E}_{4}^{\sigma}\left(\hat{E}_{2}^{\rho}\right)^{2}-29 \hat{E}_{4}^{\rho} \hat{E}_{4}^{\sigma}\right] \\
& +\mathcal{O}\left(\nu^{6}\right) .
\end{aligned}
$$

Note that the conjugacy class represented by $\alpha^{n_{1}} \beta^{m_{1}} \alpha^{n_{2}} \beta^{m_{2}}$ is the same if one interchanges $\left(n_{1}, m_{1}\right)$ with $\left(n_{2}, m_{2}\right)$. Furthermore, if $\left(n_{1}, m_{1}\right)=\left(n_{2}, m_{2}\right)$, this is not a primitive class and should not be included in the infinite product definition of $\mathcal{F}(\Omega)$. Putting these together,

$$
\begin{aligned}
\prod_{m=1}^{\infty} \prod_{\gamma \neq \alpha^{ \pm 1}, \beta^{ \pm 1}, \text { prim.cl. }}\left(1-q_{\gamma}^{m}\right)= & -(2 \pi i \nu)^{2} 4 \hat{E}_{2}^{\rho} \hat{E}_{2}^{\sigma}+\frac{(2 \pi i \nu)^{4}}{3} \\
\times & {\left[4\left(\hat{E}_{2}^{\rho}\right)^{2} \hat{E}_{2}^{\sigma}+4\left(\hat{E}_{2}^{\sigma}\right)^{2} \hat{E}_{2}^{\rho}-\hat{E}_{4}^{\rho} \hat{E}_{2}^{\sigma}-\hat{E}_{4}^{\sigma} \hat{E}_{2}^{\rho}\right.} \\
& \left.+20 \hat{E}_{4}^{\rho}\left(\hat{E}_{2}^{\sigma}\right)^{2}+20 \hat{E}_{4}^{\sigma}\left(\hat{E}_{2}^{\rho}\right)^{2}-5 \hat{E}_{4}^{\rho} \hat{E}_{4}^{\sigma}\right] \\
& +\mathcal{O}\left(\nu^{6}\right),
\end{aligned}
$$




$$
\begin{aligned}
\prod_{m=1}^{\infty}\left(1-q_{\alpha}^{m}\right)= & \prod_{m=1}^{\infty}\left(1-e^{2 \pi i m \rho}\right)\left[1+(2 \pi i \nu)^{2} 2 \hat{E}_{2}^{\rho} \hat{E}_{2}^{\sigma}+(2 \pi i \nu)^{4}\right. \\
& \times\left(-\frac{2}{3}\left(\hat{E}_{2}^{\rho}\right)^{2} \hat{E}_{2}^{\sigma}-\frac{1}{3} \hat{E}_{2}^{\rho}\left(\hat{E}_{2}^{\sigma}\right)^{2}+6\left(\hat{E}_{2}^{\rho}\right)^{2}\left(\hat{E}_{2}^{\sigma}\right)^{2}\right. \\
& \left.\left.+\frac{1}{6} \hat{E}_{2}^{\rho} \hat{E}_{4}^{\sigma}-\frac{10}{3}\left(\hat{E}_{2}^{\rho}\right)^{2} \hat{E}_{4}^{\sigma}-\frac{5}{3} \hat{E}_{4}^{\rho}\left(\hat{E}_{2}^{\sigma}\right)^{2}\right)+\mathcal{O}\left(\nu^{6}\right)\right]
\end{aligned}
$$

Finally, we arrive at the expansion for $\mathcal{F}(\Omega)$,

$$
\begin{aligned}
\frac{\mathcal{F}(\Omega)}{\prod_{m=1}^{\infty}\left(1-q^{m}\right)^{2}\left(1-s^{m}\right)^{2}}= & 1+(2 \pi i \nu)^{2} 4 \hat{E}_{2}^{\rho} \hat{E}_{2}^{\sigma}+\frac{(2 \pi i \nu)^{4}}{3} \\
& \times\left[-2\left(\hat{E}_{2}^{\rho}\right)^{2} \hat{E}_{2}^{\sigma}-2 \hat{E}_{2}^{\rho}\left(\hat{E}_{2}^{\sigma}\right)^{2}+48\left(\hat{E}_{2}^{\rho}\right)^{2}\left(\hat{E}_{2}^{\sigma}\right)^{2}\right. \\
& \left.-10\left(\hat{E}_{2}^{\rho}\right)^{2} \hat{E}_{4}^{\sigma}-10\left(\hat{E}_{2}^{\sigma}\right)^{2} \hat{E}_{4}^{\rho}-5 \hat{E}_{4}^{\rho} \hat{E}_{4}^{\sigma}\right]+\mathcal{O}\left(\nu^{6}\right)
\end{aligned}
$$

\section{Appendix D. The sewing parameters}

The holomorphic correction factor $G(\Omega)$ has an expansion in $\epsilon$ up to order $\epsilon^{4}$ as

$$
G(\Omega)=1-\epsilon^{2} \frac{E_{2}\left(\tau_{1}\right) E_{2}\left(\tau_{2}\right)}{72}
$$

$$
+\epsilon^{4}\left[\frac{E_{2}\left(\tau_{1}\right)^{2} E_{2}\left(\tau_{2}\right)^{2}}{6912}+\frac{E_{4}\left(\tau_{1}\right) E_{4}\left(\tau_{2}\right)}{17280}\right]+\mathcal{O}\left(\epsilon^{6}\right) .
$$

The $\mathcal{O}\left(\epsilon^{2}\right)$ result was given in [5]. The $\mathcal{O}\left(\epsilon^{4}\right)$ result is obtained by comparing the order $k$ term in $Z_{\text {fake }}$ with our conjectured expression for $S_{0}$, and further verified by comparing with the factorization of the genus two partition functions of $k=1,2,3$ ECFTs. The sewing parameters $\tau_{1}, \tau_{2}, \epsilon$ of [5] are related 
to the period matrix elements $\rho, \sigma, \nu$ by

(D.2)

$$
\begin{aligned}
& e^{2 \pi i \rho}=e^{2 \pi i \tau_{1}}\left\{1-\epsilon^{2} \frac{E_{2}\left(\tau_{2}\right)}{12}+\epsilon^{4} E_{2}\left(\tau_{2}\right)^{2}\left[\frac{1}{288}-\frac{E_{2}\left(\tau_{1}\right)}{1728}\right]+\mathcal{O}\left(\epsilon^{6}\right)\right\} \\
& e^{2 \pi i \sigma}=e^{2 \pi i \tau_{2}}\left\{1-\epsilon^{2} \frac{E_{2}\left(\tau_{1}\right)}{12}+\epsilon^{4} E_{2}\left(\tau_{1}\right)^{2}\left[\frac{1}{288}-\frac{E_{2}\left(\tau_{2}\right)}{1728}\right]+\mathcal{O}\left(\epsilon^{6}\right)\right\} \\
& 2 \pi i \nu=\epsilon\left[1+\epsilon^{2} \frac{E_{2}\left(\tau_{1}\right) E_{2}\left(\tau_{2}\right)}{144}+\mathcal{O}\left(\epsilon^{4}\right)\right]
\end{aligned}
$$

Or inversely,

(D.3)

$$
\begin{aligned}
e^{2 \pi i \tau_{1}}= & e^{2 \pi i \rho}\left\{1+(2 \pi i \nu)^{2} \frac{E_{2}(\sigma)}{12}+(2 \pi i \nu)^{4}\left[\frac{E_{2}(\sigma)^{2}}{288}-\frac{E_{2}(\rho) E_{4}(\sigma)}{1728}\right]\right. \\
& \left.+\mathcal{O}\left(\nu^{6}\right)\right\}, \\
e^{2 \pi i \tau_{2}}= & e^{2 \pi i \sigma}\left\{1+(2 \pi i \nu)^{2} \frac{E_{2}(\rho)}{12}+(2 \pi i \nu)^{4}\left[\frac{E_{2}(\rho)^{2}}{288}-\frac{E_{2}(\sigma) E_{4}(\rho)}{1728}\right]\right. \\
& \left.+\mathcal{O}\left(\nu^{6}\right)\right\}, \\
\epsilon= & 2 \pi i \nu\left[1-(2 \pi i \nu)^{2} \frac{E_{2}(\rho) E_{2}(\sigma)}{144}+\mathcal{O}\left(\nu^{4}\right)\right] .
\end{aligned}
$$

As pointed out in [5], the genus two partition function $Z_{k, g=2}$ that naturally factorizes into one-point functions on tori with moduli $\tau_{1}, \tau_{2}$, and sewed together using the pinching parameter $\epsilon$ is not the modular partition function $Z_{k, g=2}^{\text {mod }}$. Rather, it is related to $Z_{k, g=2}^{\bmod }$ by

$$
Z_{k, g=2}(\Omega)=\frac{Z_{k, g=2}^{\bmod }(\Omega)}{G(\Omega)^{k}}
$$

Near the separating degeneration, one has

(D.5)

$$
\begin{aligned}
Z_{k, g=2}(\Omega)= & \sum_{i} \epsilon^{-2 k+\Delta_{i}}\left\langle\mathcal{A}_{i}\right\rangle_{\tau_{1}}\left\langle\mathcal{A}_{i}\right\rangle_{\tau_{2}} \\
= & \frac{Z_{k, g=1}\left(\tau_{1}\right) Z_{k, g=1}\left(\tau_{2}\right)}{\epsilon^{2 k}}+\frac{\frac{1}{2 \pi i} \partial_{\tau} Z_{k, g=1}\left(\tau_{1}\right) \frac{1}{2 \pi i} \partial_{\tau} Z_{k, g=1}\left(\tau_{2}\right)}{12 k \epsilon^{2 k-2}} \\
& +\frac{5}{24 k(60 k+11) \epsilon^{2 k-4}}\langle T * T\rangle_{\tau_{1}}\langle T * T\rangle_{\tau_{2}}+\mathcal{O}\left(\epsilon^{6-2 k}\right),
\end{aligned}
$$


where the second term comes from the torus one-point function of the stress energy tensor, which is the only operator of dimension 2 in an ECFT. In general, for a primary field $\mathcal{O}$ of dimension $\Delta$, the torus one-point function $\langle\mathcal{O}\rangle_{\tau}$ is a weight $\Delta$ cusp form in an ECFT. In particular, $\langle\mathcal{O}\rangle_{\tau}=0$ for $\Delta<12$, and $\langle\mathcal{O}\rangle_{\tau}$ can only contribute to (D.5) starting at order $\epsilon^{12-2 k}$. At dimension 4, the Virasoro descendants are $\partial^{2} T$ and $T * T$, only the latter having a non-zero torus one-point function. To calculate $\langle T * T\rangle_{\tau}$, one can first perform a conformal transformation mapping $T * T$ from the cylinder to the complex plane $\left(z=e^{i w}\right)$,

(D.6)

$$
T * T=\oint_{C_{2}} \frac{d w_{2}}{2 \pi} \oint_{C_{1}} \frac{d w_{1}}{2 \pi i} \frac{T\left(w_{1}\right) T\left(w_{2}\right)}{w_{1}-w_{2}}
$$

$$
=\oint_{C_{2}} \frac{d z_{2}}{2 \pi i z_{2}} \oint_{C_{1}} \frac{d z_{1}}{2 \pi i z_{1}} \frac{\left(z_{1}^{2} T\left(z_{1}\right)-k\right)\left(z_{2}^{2} T\left(z_{2}\right)-k\right)}{\ln z_{1}-\ln z_{2}}
$$

where the contour $C_{1}$ goes around $w_{2}$ or $z_{2}$. On the $z$-plane, one can compute the operator corresponding to $T * T, \mathcal{O}_{T * T}$, by computing the integral on the RHS of (D.6). $C_{1}$ can be chosen as the sum of a contour inside $C_{2}$ clockwise around $z=0$, and another counterclockwise contour outside $C_{2}$. We end up with

$$
\mathcal{O}_{T * T}=\left(L_{0}-k\right)^{2}-\frac{L_{0}-k}{6}+\frac{k}{60}+2 \sum_{n=1}^{\infty} L_{-n} L_{n} .
$$

Equation (4.12) follows from the trace of (D.7).

\section{Appendix E. Partition functions on hyperelliptic curves}

In this appendix, we recall some properties of the partition function of a CFT with $c=24 k$ on a genus $g$ hyperelliptic curve $y^{2}=\prod_{i=1}^{2 g+2}\left(x-e_{i}\right)$, following [1]. We can write

$$
\tilde{Z}\left(e_{1}, \ldots, e_{2 g+2}\right)=\left[\prod_{1 \leq i<j \leq 2 g+2}\left(e_{i}-e_{j}\right)^{k}\right]\left\langle\mathcal{E}\left(e_{1}\right) \cdots \mathcal{E}\left(e_{2 g+2}\right)\right\rangle
$$


where $\mathcal{E}$ is the twist field in the 2-fold symmetric product CFT (on the $x$-plane). Under the $\mathrm{SL}(2, \mathbf{C})$ action,

$$
\begin{aligned}
& e_{i} \longrightarrow \frac{a e_{i}+b}{c e_{i}+d}, \\
& \tilde{Z} \longrightarrow \tilde{Z} \prod_{i=1}^{2 g+2}\left(c e_{i}+d\right)^{(2-2 g) k} .
\end{aligned}
$$

The $e_{i}$ 's are determined by the moduli of the hyperelliptic Riemann surface up to the overall $\mathrm{SL}(2, \mathbf{C})$ action and permutations. To construct an $\mathrm{SL}(2, \mathbf{C})$ invariant expression, consider the differential

$$
\Theta=\frac{d x}{y} \wedge \frac{x d x}{y} \wedge \cdots \wedge \frac{x^{g-1} d x}{y},
$$

regarded as a top form on the space of holomorphic 1-forms. Under $\mu, x$ and $y$ transform as

$$
x \longrightarrow \frac{a x+b}{c x+d}, \quad y \longrightarrow \frac{y}{(c x+d)^{g+1} \prod_{i}\left(c e_{i}+d\right)^{1 / 2}} .
$$

As a consequence, (E.3) gets multiplied by $\prod_{i}\left(c e_{i}+d\right)^{g / 2}$. The partition function, as a function of the period matrix $\Omega$ and invariant under $\operatorname{SL}(2, \mathbf{C})$, can be recovered from (E.1),

$$
Z(\Omega) \propto\left(\int_{\alpha^{1} \wedge \cdots \wedge \alpha^{g}} \Theta\right)^{(4-4 / g) k} \tilde{Z}\left(e_{1}, \ldots, e_{2 g+2}\right),
$$

where the integral is understood to be on $\bigwedge^{g} H^{1}\left(\Sigma_{g}\right)$. The modular group $\mathrm{Sp}(2 g, \mathbf{Z})$ acts as monodromies on the $e_{i}$ 's, in general permuting them; it also acts on the basis 1 -cycles, $\alpha^{I}, \beta_{I}, I=1, \ldots, g$. We can choose a set of holomorphic 1-forms $\omega_{I}$, with

$$
\int_{\alpha^{I}} \omega_{J}=\delta_{J}^{I}, \quad \int_{\beta_{I}} \omega_{J}=\Omega_{I J}
$$

Under $\operatorname{Sp}(2 g, \mathbf{Z})$, they transform as

$$
\begin{aligned}
\alpha \longrightarrow D \alpha+C \beta, \quad \beta \longrightarrow B \alpha+A \beta, & \longrightarrow\left((C \Omega+D)^{-1}\right)_{I}{ }^{J} \omega_{J}, \quad \Omega \longrightarrow(A \Omega+B)(C \Omega+D)^{-1} .
\end{aligned}
$$

Therefore (E.3) transforms with a Jacobian factor $\operatorname{det}(C \Omega+D)^{-1}$, and $Z(\Omega)$ transforms as an $\operatorname{Sp}(2 g, \mathbf{Z})$ modular form with weight $w=(4-4 / g) k$. 
For $g>2$, it is more natural to define the partition function $T(\Omega)$ to have weight $12 k$ and be free of singularities. In the hyperelliptic case, we can write

$$
\widetilde{T}\left(e_{1}, \ldots, e_{2 g+2}\right)=\left[\prod_{1 \leq i<j \leq 2 g+2}\left(e_{i}-e_{j}\right)^{3 k}\right]\left\langle\mathcal{E}\left(e_{1}\right) \cdots \mathcal{E}\left(e_{2 g+2}\right)\right\rangle,
$$

and similarly

$$
T(\Omega) \propto\left(\int_{\alpha^{1} \wedge \ldots \wedge \alpha^{g}} \Theta\right)^{12 k} \tilde{T}\left(e_{1}, \ldots, e_{2 g+2}\right)
$$

$\tilde{T}$ has weight $-6 g k$ with respect to the $\operatorname{SL}(2, \mathbf{C})$ action, leading to $T(\Omega)$ of weight $12 k$ under $\operatorname{Sp}(2 g, \mathbf{Z})$.

A useful result is the partition function of a chiral boson $\phi$ on the hyperelliptic curve [18], $\tilde{T}_{\phi}\left(e_{1}, \ldots, e_{2 g+2}\right)=\left(\int_{\alpha^{1} \wedge \cdots \wedge \alpha^{g}} \Theta\right)^{-1 / 2}$, and hence $T_{\phi}(\Omega)=1$. In the case of genus one and two, this is equivalent to the well known results $Z_{\phi, g=1}(\tau)=\eta(\tau)^{-1}, Z_{\phi, g=2}(\Omega)=\chi_{10}(\Omega)^{-1 / 24}$.

The fake CFT partition functions, $Z_{\text {fake }}$ (corresponding to $Z(\Omega)$ ), or $\hat{Z}_{\text {fake }}$ (corresponding to $T(\Omega)$ ), can be defined on a hyperelliptic Riemann surface analogously. One arranges the $e_{i}$ 's in pairs $\left(e_{2 s-1}, e_{2 s}\right)$ and keeps only the Virasoro descendants in the $\mathcal{E}\left(e_{2 s-1}\right) \mathcal{E}\left(e_{2 s}\right)$ OPE. The formulae (E.5) and (E.9) generalize to the fake partition functions as well, with the difference being that $\tilde{Z}_{\text {fake }}\left(e_{1}, \ldots, e_{2 g+2}\right)$ or $\tilde{\hat{Z}}_{\text {fake }}\left(e_{1}, \ldots, e_{2 g+2}\right)$ is not invariant under the monodromies on the $e_{i}$ 's; in general they have branch cuts.

\section{References}

[1] E. Witten, Three-dimensional gravity revisited, Preprint, 2007, arXiv:0706.3359 [hep-th].

[2] I. Frenkel, J. Lepowsky and A. Meurman, A natural representation of the Fischer-Griess Monster with the modular function $J$ as character, Proc. Natl. Acad. Sci. USA 81 (1984), 3256-3260; Vertex Operator Algebras and the Monster, Boston, USA: Academic (1988) 508 P. (Pure and Applied Mathematics, 134)

[3] D. Gaiotto and X. Yin, Genus two partition functions of extremal conformal field theories, Preprint, 2007, arXiv:0707.3437 [hep-th]. 
[4] M.R. Gaberdiel, Constraints on extremal self-dual CFTs, Preprint, 2007, arXiv:0707.4073 [hep-th].

[5] M.P. Tuite, Genus two meromorphic conformal field theory, Preprint, 2007, arXiv:math.qa/9910136.

[6] J.M. Maldacena and A. Strominger, AdS(3) black holes and a stringy exclusion principle, JHEP 9812 (1998), 005, arXiv:hep-th/9804085.

[7] A. Maloney and E. Witten, Quantum gravity partition functions in three dimensions, arXiv:0712.0155 [hep-th].

[8] J. Manschot, $A d S_{3}$ partition functions reconstructed, Preprint, 2007, arXiv:0707.1159 [hep-th].

[9] K. Krasnov, Holography and Riemann surfaces, Adv. Theor. Math. Phys. 4, 929 (2000), 929, arXiv:hep-th/0005106.

[10] P.G. Zograf and L.A. Takhtajan, On the uniformization of Riemann surfaces and on the Weil-Petersson metric on the Teichmüller and Schottky Spaces, Math.USSR-Sb. 60 (1988), 297-313.

[11] A. McIntyre and L.A. Takhtajan, Holomorphic factorization of determinants of laplacians on Riemann surfaces and a higher genus generalization of Kronecker's first limit formula, Preprint, 2004, arXiv:math/0410294.

[12] Y. Leblanc, The genus 2 free energy of the closed bosonic string, Phys. Rev. D 39 (1989), 3731.

[13] O. Coussaert, M. Henneaux and P. van Driel, The asymptotic dynamics of three-dimensional Einstein gravity with a negative cosmological constant, Class. Quant. Grav. 12 (1995), 2961, arXiv:gr-qc/9506019.

[14] L.A. Takhtajan and L.P. Teo, Quantum Liouville theory in the background field formalism. I: Compact Riemann surfaces, Commun. Math. Phys. 268 (2006), 135, arXiv:hep-th/0508188.

[15] J.-I. Igusa, On siegel modular forms of genus two, Amer. J. Math. 84 (1962), 175-200; Modular forms and projective invariants, Amer. J. Math. 89 (1967), 817-855.

[16] D. Friedan and S.H. Shenker, The analytic geometry of two-dimensional conformal field theory, Nucl. Phys. B 281 (1987) 509. 
[17] R. Dijkgraaf, J.M. Maldacena, G.W. Moore and E.P. Verlinde, A black hole farey tail, Preprint, 2005, arXiv:hep-th/0005003.

[18] A. B. Zamolodchikov, Conformal scalar field on the hyperelliptic curve and critical ashkin-teller multipoint correlation functions, Nucl. Phys. B 285, (1987), 481.

JefFerson Physical Laboratory

HARVARD UNIVERSITY

CAMBRIDGE

MA 02138

USA

E-mail address: xiyin@fas.harvard.edu

Received OCtober 14, 2007 